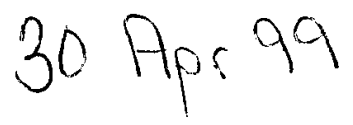

\title{
MODELING THE EFFECTS OF HOLE DISTRIBUTION IN \\ PERFORATED ALUMINUM SHEETS I : REPRESENTATIVE UNIT CELLS
}

\author{
G. F. Raiser, S. Jia, and G. L. Povirk \\ Department of Mechanical Engineering \\ Yale University \\ New Haven, Connecticut 06520 USA
}

\begin{abstract}
A method is presented that incorporates microstructural information into a model of the mechanical behavior of two-phase composite materials. The approach is to determine periodic microstructures that are statistically similar to the actual microstructure of the material under consideration. The utility of this method is that computationally tractable finite element simulations can then be carried out on representative unit cells that are directly obtained from microstructural observations. To illustrate this method, mechanical tests are performed on perforated aluminum sheets with various microstructures, and the results are compared to finite element simulations of selected representative unit cells. The simulations agree with the trends observed in the experiments, including measurements of the overall strength and ductility of the sheets. Advantages and limitations of the approach used here are discussed.
\end{abstract}

\section{DISTRIBUTION STATEMENT A Approved for Public Release Distribution Unlimited}




\section{REPORT DOCUMENTATION PAGE}

Public reporting burden for this collection of information is estimated to average 1 hour per response, including gathering and maintaining the data needed, and completing and reviewing the collection of information. Send $c$

mashington Headquarters Services,

collection of intormation, including suggestions for reducing this burden,
Davis Highway, Suite 1204. Arlington. VA 22202-4302, and to the Office of Management and Budget, Paperwork Red

1. AGENCY USE ONLY (Leave 2. REPORT DATE Blank) $9 / 12 / 00$

1. . Final, $5 / 1 / 96-4 / 30 / 99$

4. TITLE AND SUBTITLE

Modeling and Process Control of Composite Microstructures

\section{AFRL-SR-BL-TR-00-}

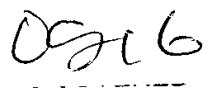

5. FUNDING NUMBERS

F49960-96-1-0198

6. AUTHORS

Gary L. Povirk

7. PERFORMING ORGANIZATION NAME(S) AND ADDRESS(ES)

Yale University

P.O. Box 208337

New Haven, CT 06520-8337

9. SPONSORING / MONITORING AGENCY NAME(S) AND ADDRESS(ES)

Air Force Office of Scientific Research

110 Duncan Avenue, Room B115

Bolling AFB, DC 20332-8050

8. PERFORMING ORGANIZATION REPORT NUMBER

10. SPONSORING / MONITORING AGENCY REPORT NUMBER

11. SUPPLEMENTARY NOTES

12a. DISTRIBUTION / AVAILABILITY STATEMENT

12b. DISTRIBUTION CODE

UL

13. ABSTRACT (Maximum 200 words)

See final report

\section{4}

14. SUBJECT TERMS

17. SECURITY CLASSIFICATION OF REPORT (

unclassified

NSN 7540-01-280-5500
15. NUMBER OF PAGES

16. PRICE CODE

20. LIMITATION OF ABSTRACT

UL

Standard Form 298 (Rev. 2-89) Prescribed by ANSI Std. Z39-1 298-102 


\section{Introduction}

Materials with two distinct phases represent a broad class of materials, including dispersionstrengthened alloys, mechanically-alloyed materials, spheroidized steels, and metal- and ceramicmatrix composites. Incomplete densification during processing, creep cavitation, or void nucleation during ductile fracture can cause even nominally homogeneous materials to contain a significant fraction of voids. These voids can also be thought of as secondary phase. The microstructure of a two-phase material can in turn have a profound effect on the mechanical properties of the material, particularly on the initiation and evolution of damage. For example, research by Lewandowski et al. [1] on aluminum-silicon carbide composites has shown that fracture usually initiates at large particles, inclusions, and regions of clustered silicon carbide particles. They also observed that damage accumulation ahead of a macroscopic crack tends to occur in regions where reinforcements are clustered together.

Many investigators have studied two-phase materials by idealizing the composite microstructure as having a simple periodic structure, with one or two reinforcing particles (or voids) within each unit cell. With this assumption, estimates of the macroscopic response of the material can be obtained by solving an appropriate boundary value problem formulated on a representative unit cell. This approach has been used to estimate the flow behavior of metal-matrix composites [2,3] and of porous metals [4]. Moreover, simple unit cell models have be used to study the damage mechanisms in composite materials, such as interfacial debonding [5], particle cracking [6], and ductile failure in the matrix [7]. While these analyses have provided important insights into deformation and damage initiation in these materials, they also possess significant limitations as well. One important limitation is that the predicted behavior can be strongly affected by the choice of assumed unit cell parameters $[2,8]$. Furthermore, a simple unit cell with one reinforcement (or void) cannot provide a realistic portrayal of damage evolution in a composite material. For example, experiments on aluminum sheets containing randomly distributed perforations showed the pronounced effects of hole distribution on resultant mechanical behavior [9]. Corresponding finite element calculations by Becker and Smelser [10] showed that a simple unit cell with one hole could not adequately predict the ductility of the sheets. 
More recently, investigators have begun to account for the effects of microstructure on the mechanical properties of two-phase materials. A central problem that develops, however, is the computational expense associated with incorporating large amounts of microstructural information into the model. To make the problems more tractable, researchers have used different procedures to simplify the problem. For example, Ostoja-Starzewski et al. [11] developed a finite difference lattice model in which damage evolution is simulated by sequentially removing bonds in the model if the lattice strain exceeds a critical value. While this method is quite useful in examining the effects of material constants on the qualitative behavior of composite materials, more detailed descriptions of material behavior are required to model real composite materials. Ghosh et al. [12] has developed a model in which a composite material is divided into Voronoi cells, with each cell containing one reinforcement at most. For each Voronoi cell, a stress hybrid method is used where an equilibrated stress field (derived from an Airy stress function) is assumed within the cell and displacements are interpolated on the boundary of the cell. While the method is complex, it appears to have great potential in modeling deformation and damage within composite materials.

The intent of this work is to develop alternative tools for incorporating microstructural information into models of two-phase materials. The approach considered here is to determine a periodic microstructure that is statistically similar to the actual microstructure under consideration. With a periodic microstructure so determined, a numerical analysis can be performed on a single unit cell of the periodic structure, with an associated reduction in computational cost. To test the procedures, idealized composites are constructed by perforating aluminum sheets with various complex patterns of holes and subjecting the specimens to standard uniaxial tensile tests. After corresponding periodic microstructures have been determined, finite element simulations of uniaxial tensile tests are performed on representative unit cells and the results are compared to experimental results. This study is a continuation of an investigation by Povirk [13], who compared the elastic behavior of complex microstructures to that of selected representative unit cells.

\section{Procedures}

The general procedures used in this study are outlined as follows. Six complex patterns of circles were generated and used as templates to obtain aluminum sheets with various hole distributions. 
The mechanical behavior of the perforated sheets was then determined experimentally by uniaxial tensile testing. Numerical models of the six complex microstructures were developed by first obtaining corresponding unit cells that have similar spatial distributions of holes; the methods used in the determination of the unit cell parameters will be discussed subsequently. Finite element meshes were then generated for each of the six unit cells, and appropriate periodic boundary conditions were implemented. The flow behavior of the aluminum matrix was characterized by isotropic, rate-dependent $J_{2}$ flow theory, with initial strain-hardening followed by eventual strainsoftening. The behavior predicted by the unit cell models were then compared to the corresponding experimental results.

\subsection{Generation of Hole Patterns}

The region in which the hole patterns were generated were assumed to occupy a rectangular region of dimensions $H_{1} \times H_{2}$ (see Figure 1). Each pattern was arbitrarily assumed to contain 115 holes, with the hole diameter chosen such that the area fraction was 10 percent. The initial step was to discretize this region into an $N_{1} \times N_{2}$ array of points, given by

$$
x_{k_{1}}=\frac{H_{1}}{N_{1}} k_{1}, \quad k_{1}=0, \ldots, N_{1} \quad ; \quad y_{k_{1}}=\frac{H_{2}}{N_{\dot{2}}} k_{2}, \quad k_{2}=0, \ldots, N_{2}
$$

The hole patterns were generated by assigning, at each of the discrete points defined by equation (1), a number $p$ of the form

$$
\begin{aligned}
p & =r+\varepsilon_{1} \cos \left(\frac{12 \pi x}{H_{1}}\right)+\varepsilon_{2} \cos \left(\frac{12 \pi y}{H_{2}}\right) \\
& +\frac{\varepsilon_{3}}{2}\left[\sin \left(\frac{12 \pi x}{H_{1}}-\frac{\pi}{2}\right) \sin \left(\frac{12 \pi y}{H_{2}}-\frac{\pi}{2}\right)+1\right] \\
& +\frac{\varepsilon_{4}}{2}\left[\sin \left(\frac{12 \pi x}{H_{1}}-\frac{\pi}{2}\right) \sin \left(\frac{6 \pi y}{H_{2}}-\frac{\pi}{2}\right)+1\right] \\
& +\frac{\varepsilon_{5}}{2}\left[\sin \left(\frac{6 \pi x}{H_{1}}-\frac{\pi}{2}\right) \sin \left(\frac{12 \pi y}{H_{2}}-\frac{\pi}{2}\right)+1\right]
\end{aligned}
$$

where $r$ represents a random number ranging from 0 to 1 , and the variables $\varepsilon_{i}$ are input parameters that were used to exert a measure of control over the generated patterns of holes. Circles were 
placed starting at the point with the maximum value of $p$ and sequentially thereafter at points with descending values of $p$. Any new circle location that overlapped or touched an existing circle was rejected. Six different microstructures were generated by selecting an initial "seed" for the random number generator and choosing different values of the parameters $\varepsilon_{i}$. Templates for the aluminum sheets were generated based on the calculated hole coordinates.

\subsection{Experimental Procedure}

Specimens were machined from 1100-H14 aluminum sheets. Each sample has a thickness of 0.81 $\mathrm{mm}$, a width of $30.5 \mathrm{~mm}$ and a gauge length of $30.5 \mathrm{~mm}$. The hole patterns were printed out on a laser printer and bonded to the aluminum tensile samples. With the template attached to the specimens, holes with a diameter of $1.016 \mathrm{~mm}$ were drilled in the appropriate locations. For each of the six patterns that were generated, three identical samples were prepared. After drilling, any excessive metal extending out of the holes was sanded off, and the specimens were subjected to annealing at $400^{\circ} \mathrm{C}$ for $60 \mathrm{~s}$. (O-temper [14]).

Tensile tests were conducted on an MTS servo-hydraulic testing machine configured with a 458.20 MicroConsole using a TestLink interface. The MicroConsole was equipped with a DC controller for control and data acquisition of the loads and an $\mathrm{AC}$ controller for control and data acquisition of the displacement. A MTS 632.11B-20 extensometer with an attachment kit for flat sample applied was used to measure the strain of the sample. The default distance between the two knife edges of the extensometer was $25.4 \mathrm{~mm}$ but was modified to be $30.5 \mathrm{~mm}$ to match the gauge length of the sample. Another DC controller on the Microconsole was used for control and data acquisition of the strain data from the extensometer.

One difficulty involved in the experiments was that as failure progressed, significant rotation of the specimen would often occur. This is an important problem, since the model of sheet deformation does not account for this effect. To limit this effect, we first applied a small tensile load to align the sample. Once the specimen is aligned, we then tightened two adjustable "stops" on each side of specimen to limit the rotation of the specimen during testing. The extensometer was then attached to the sample, and a constant velocity of $0.04 \mathrm{~mm} / \mathrm{s}$ was prescribed to the lower grip. The load, displacement and strain (actually distance between two knife edges of the extensometer) data was 
output to a computer through a LAB-PC data acquisition expansion board. A LabVIEW [15] program was created to process these data and output the engineering stress and strain of the sample. Each sample was pulled to complete failure.

\subsection{Selection of Unit Cell Parameters}

The first step in the selection of unit cell parameters is to represent a complex pattern of holes by a discrete indicator function

$$
g^{c}\left(x_{k_{1}}, y_{k_{2}}\right)= \begin{cases}0 & \text { for phase } 1 \\ 1 & \text { for phase } 2\end{cases}
$$

where phase 1 and phase 2 represents the aluminum matrix and the holes, respectively. The corresponding unit cell that will be used for the finite element calculations is described by a similar indicator function.

The power spectral density of the complex patterns is obtained at discrete frequencies by first calculating the two-dimensional, discrete, fast Fourier transform of the indicator function, $G^{c}\left(f_{x}^{n_{1}}, f_{y}^{n_{2}}\right)$

$$
G^{c}\left(f_{x}^{n_{1}}, f_{y}^{n_{2}}\right)=\sum_{k_{2}=0}^{N_{2}-1} \sum_{k_{1}=0}^{N_{1}-1} \exp \left(2 \pi i x_{k_{1}} f_{x}^{n_{1}}\right) \exp \left(2 \pi i y_{k_{2}} f_{y}^{n_{2}}\right) g^{c}\left(x_{k_{1}}, y_{k_{2}}\right)
$$

at frequencies given by

$$
f_{x}^{n_{1}}=\frac{n_{1}}{H_{1}}, \quad n_{1}=-\frac{N_{1}}{2}, \ldots, \frac{N_{1}}{2} \quad f_{y}^{n_{2}}=\frac{n_{2}}{H_{2}}, \quad n_{2}=-\frac{N_{2}}{2}, \ldots, \frac{N_{2}}{2}
$$

Because the indicator function is real, its Fourier transform is independent of the signs of the frequencies $f_{x}^{n_{1}}$ and $f_{y}^{n_{2}}$. The one-sided power spectral density of the complex pattern, $P^{c}\left(f_{x}^{n_{1}}, f_{y}^{n_{2}}\right)$, can then be written as [16]

$$
P^{c}\left(f_{x}^{n_{1}}, f_{y}^{n_{2}}\right)= \begin{cases}\left|G^{c}\left(f_{x}^{n_{1}}, f_{y}^{n_{2}}\right)\right|^{2} & \text { for } n_{1}, n_{2}=0 \\ 2\left|G^{c}\left(f_{x}^{n_{1}}, f_{y}^{n_{2}}\right)\right|^{2} & \text { for } n_{1}>0, n_{2}=0, \text { or } n_{1}=0, n_{2}>0 \\ 4\left|G^{c}\left(f_{x}^{n_{1}}, f_{y}^{n_{2}}\right)\right|^{2} & \text { for } n_{1}, n_{2}>0\end{cases}
$$


The use of the fast Fourier transform requires that both $N_{1}$ and $N_{2}$ be powers of two. In the following discussions, frequencies with indices of $n_{1}$ and $n_{2}$ will refer to those associated with the complex hole patterns.

The next step is to choose the desired number of holes for the unit cell, an initial set of dimensions $h_{1}$ and $h_{2}$ for the cell, and an initial set of hole positions. The dimensions for the cell were chosen to yield the same area fraction of holes as in the complex patterns. The indicator function for the unit cell is then written as

$$
g^{p}(x, y)=\sum_{\beta=1}^{n_{p}} g_{0}\left(x-\phi_{x}^{\beta}, y-\phi_{y}^{\beta}\right)
$$

where $g_{0}(x, y)$ describes the shape of the holes, $\phi_{x}^{\beta}$ and $\phi_{y}^{\beta}$ are the coordinates of hole $\beta$ and $n_{p}$ is the number of holes in each unit cell. For this study, each unit cell was assumed to contain twelve holes. The unit cell is discretized by an $M_{1} \times M_{2}$ array of points so that the fast Fourier transform of the periodic structure then takes the form

$$
G^{p}\left(f_{x}^{m_{1}}, f_{y}^{m_{2}}\right)=\sum_{\beta=1}^{n_{p}} G_{0}\left(f_{x}^{m_{1}}, f_{y}^{m_{2}}\right) \exp \left(2 \pi i f_{x}^{m_{1}} \phi_{x}^{\beta}\right) \exp \left(2 \pi i f_{y}^{m_{2}} \phi_{y}^{\beta}\right)
$$

at frequencies given by

$$
f_{x}^{m_{1}}=\frac{m_{1}}{h_{1}}, \quad m_{1}=-\frac{M_{1}}{2}, \ldots, \frac{M_{1}}{2}, \quad f_{y}^{m_{2}}=\frac{m_{2}}{h_{2}}, \quad m_{2}=-\frac{M_{2}}{2}, \ldots, \frac{M_{2}}{2}
$$

and where $G_{0}\left(f_{x}^{m_{1}}, f_{y}^{m_{2}}\right)$ represents the fast Fourier transform of $g_{0}(x, y)$. The corresponding power spectral density can be obtained by equation (6). Frequencies with indices of $m_{1}$ and $m_{2}$ will always refer to those associated with the unit cell.

Note that the discrete power spectral densities of the complex and periodic structures are, in general, obtained at different frequencies. Furthermore, assuming that the dimensions of the unit cell are smaller than that of the random structure, the frequencies associated with the periodic structure will be spaced at larger intervals in comparison to the complex structure. Therefore, to directly compare the spectral density of each structure, the power spectral density of the complex structure has to be "rebinned" to match the frequencies of the periodic structure. For a frequency bin defined by 


$$
\frac{f_{x}^{m_{1}-1}+f_{x}^{m_{1}}}{2}<f_{x}<\frac{f_{x}^{m_{1}}+f_{x}^{m_{1}+1}}{2}, \quad \frac{f_{y}^{m_{2}-1}+f_{y}^{m_{2}}}{2}<f_{y}<\frac{f_{y}^{m_{2}}+f_{y}^{m_{2}+1}}{2}
$$

the rebinned power spectral density of the complex microstructure $\hat{P}^{c}\left(f_{x}^{m_{1}}, f_{y}^{m_{2}}\right)$ is written as

$$
\hat{P}^{c}\left(f_{x}^{m_{1}}, f_{y}^{m_{2}}\right)=\sum_{n_{2}=n_{2}^{i}}^{n_{2}^{f}} \sum_{n_{1}=n_{1}^{i}}^{n_{1}^{f}} w\left(n_{1}, n_{2}\right) P^{c}\left(f_{x}^{n_{1}}, f_{y}^{n_{2}}\right)
$$

where $n_{1}^{i} \leq n_{1} \leq n_{1}^{f}$ and $n_{2}^{i} \leq n_{2} \leq n_{2}^{f}$ are the range of indices whose frequency bins are within the intervals given in (10). Similarly, $w\left(n_{1}, n_{2}\right)$ represents the percentage of the frequency bin of $P^{c}\left(f_{x}^{n_{1}}, f_{y}^{n_{2}}\right)$ located in the intervals in (10).

For a given set of unit cell dimensions, the hole positions are found by minimizing the function

$$
\chi^{2}=\sum_{m_{2}=0}^{M_{2} / 2} \sum_{m_{1}=0}^{M_{1} / 2}\left[\frac{\hat{P}^{c}\left(f_{x}^{m_{1}}, f_{y}^{m_{2}}\right)-\left(A^{c} / A^{p}\right) P^{p}\left(f_{x}^{m_{1}}, f_{y}^{m_{2}}\right)}{\hat{P}^{c}\left(f_{x}^{m_{1}}, f_{y}^{m_{2}}\right)}\right]^{2}+\Phi\left(\phi_{x}^{1}, \ldots, \phi_{x}^{n p}, \phi_{y}^{1}, \ldots, \phi_{y}^{n p}\right)
$$

where $A^{c}$ and $A^{p}$ are the areas of the entire structure and the unit cell respectively. The function $\Phi$ becomes large when holes are either in contact or very close to one another, thereby providing an artificial penalty for solutions that are inconsistent with the form of the indicator function given in (7). Conversely, when all the holes are isolated from one another, $\Phi$ becomes identically zero. The specific form of the function $\Phi$ does not significantly affect the results.

Finding the absolute minimum of $\chi^{2}$ for a given set of cell dimensions was complicated by the fact that the residual $\chi^{2}$ typically has many local minima. Hole positions were found by initially considering the summation shown in equation (12) truncated to include only a few of the lowest frequencies, and minimizing this function. Higher frequency terms were then added in the summation, and the function was again minimized. This process was repeated until all of the terms in the summation were included. Each minimization was carried out by use of the conjugate gradient method [16]. Several initial guesses of the hole positions were required for each set of unit cell dimensions to be reasonably assured that a reasonable correlation between the spectral densities of each microstructure was obtained. With the current state of development of the numerical procedures, convergence to the absolute minimum of $\chi^{2}$ is not guaranteed. An 
incremental search was performed over all realistic unit cell dimensions by continually adjusting the aspect ratio $h_{2} / h_{1}$. The most representative unit cell had dimensions and hole positions corresponding to the smallest value of $\chi^{2}$ obtained by the procedures.

The motivation for using the power spectral density to compare the complex and periodic hole patterns is that it can be thought of as a probability density function in the frequency domain [17]. Periodic hole patterns with similar spectral densities to that of the actual perforated sheets should therefore have a comparable hole distributions, and presumably, similar mechanical behavior as well. A more intuitive way of thinking about the power spectral density is that it can be loosely thought of as representing the "diffraction pattern" of a two-phase material, in that the spectral density of the indicator function is mathematically very similar to the intensity distribution given by the kinematical theory of electron diffraction [18]. For example, if the hole distribution is highly ordered, the spectral density will have very sharp peaks, much like the diffraction pattern of a single crystal. Conversely, the spectral density becomes more diffuse as the distribution of holes becomes more random.

\subsection{Finite Element Calculations}

Simulations of uniaxial tensile tests were performed based on the selected unit cells and the results were compared to the experimental behaviors of the corresponding aluminum sheets. Finite element meshes were obtained using the commercial mesh generating program Hypermesh [19]. Finite strain, plane stress, four-node quadrilateral elements were used in all of the computations. Periodic boundary conditions were prescribed for each unit cell to ensure compatibility with surrounding unit cells and continuity of tractions across cell boundaries. Denoting the displacement and force at the upper right corner node by $\mathbf{u}^{\mathrm{p}}$ and $\mathbf{F}^{\mathrm{p}}$ respectively, the boundary conditions were prescribed as follows. For the four corners

$$
\begin{gathered}
u_{x}(0,0)=u_{x}\left(0, h_{2}\right)=u_{y}(0,0)=u_{y}\left(h_{1}, 0\right)=0 \\
u_{x}\left(h_{1}, h_{2}\right)=u_{x}^{p}, \quad u_{y}\left(h_{1}, h_{2}\right)=u_{y}^{p}
\end{gathered}
$$

For the nodes along the edges

$$
\begin{array}{rr}
u_{x}\left(x, h_{2}\right)-u_{x}(x, 0)=0, & u_{y}\left(x, h_{2}\right)-u_{y}(x, 0)=u_{y}^{p} \\
u_{x}\left(h_{1}, y\right)-u_{x}(0, y)=u_{x}^{p}, & u_{y}\left(h_{1}, y\right)-u_{y}(0, y)=0
\end{array}
$$


To simulate uniaxial tension, a nominal strain rate of $\dot{\varepsilon}_{y 3}=2.8 \times 10^{-4} \mathrm{~s}^{-1}$ was imposed in the vertical direction, and the net forces on the sides of the unit cell were assumed to vanish. These boundary conditions are imposed through the relations

$$
\dot{u}_{y}^{p}=h_{2} \dot{\varepsilon}_{y y}, \quad F_{x}^{p}=0
$$

The nominal stress-strain behavior of the unit cell is then given by

$$
\sigma_{y y}=\frac{F_{y}^{p}}{h_{1}}, \quad \varepsilon_{y y}=\frac{u_{y}^{p}}{h_{2}}
$$

assuming an initial unit thickness. In addition to satisfying compatible cell deformations, the boundary conditions described above also yield the necessary condition that the tractions, $T$, across cell boundaries are continuous,

$$
\mathbf{T}(x, 0)=-\mathbf{T}\left(x, h_{2}\right), \quad \mathbf{T}(0, y)=-\mathbf{T}\left(h_{1}, y\right)
$$

The mechanical behavior of the aluminum was modeled as elastic-viscoplastic, with a stress ratestrain rate relation of the form

$$
\stackrel{\nabla}{\tau}=\mathbf{L}:\left(\mathbf{D}-\mathbf{D}^{p}\right)
$$

where $\tau$ is the Jaumann rate of Kirchhoff stress, $\mathbf{L}$ is a tensor of isotropic elastic moduli, and $\mathbf{D}$ is the total rate of deformation. The plastic rate of deformation was assumed to be isotropic, powerlaw viscoplasticity of the form

$$
\mathbf{D}^{p}=\dot{\varepsilon}_{0}\left(\frac{\bar{\sigma}}{g(\bar{\varepsilon})}\right)^{m} \frac{3 \mathbf{S}}{2 \bar{\sigma}}
$$

where $\bar{\sigma}$ is the effective stress, $\bar{\varepsilon}$ is the effective plastic strain, and $\mathbf{S}$ is the deviatoric stress. The flow strength $g(\bar{\varepsilon})$ of the aluminum was assumed to be first strain hardening, and then strain softening 


$$
g(\bar{\varepsilon})= \begin{cases}\sigma_{y}+\left(\sigma_{\max }-\sigma_{y}\right)\left[1-\left(\frac{\bar{\varepsilon}-\varepsilon_{1}}{\varepsilon_{1}}\right)^{2}\right]^{n}, & \bar{\varepsilon} \leq \varepsilon_{1} \\ \frac{1}{2} \sigma_{\max }\left\{1+\cos \left[\pi\left(\frac{\bar{\varepsilon}-\varepsilon_{1}}{\varepsilon_{2}-\varepsilon_{1}}\right)\right]\right\}, & \varepsilon_{1}<\bar{\varepsilon}<\varepsilon_{2} \\ 0, & \bar{\varepsilon} \geq \varepsilon_{2}\end{cases}
$$

The above relations were chosen so that the material strain-hardens up to a plastic strain of $\varepsilon_{1}$, strain-softens at plastic strains between $\varepsilon_{1}$ and $\varepsilon_{2}$, and has a complete loss of load carrying capacity at plastic strains greater than $\varepsilon_{2}$. The initial flow strength is given by $\sigma_{y}$ while the maximum flow strength is indicated by $\sigma_{\max }$. The Young's modulus of the aluminum was also assumed to be a function of accumulated plastic strain

$$
E(\bar{\varepsilon})= \begin{cases}E_{0}, & \bar{\varepsilon} \leq \varepsilon_{1} \\ \frac{1}{2} E_{0}\left\{1+\cos \left[\pi\left(\frac{\vec{\varepsilon}-\varepsilon_{1}}{\varepsilon_{2}-\varepsilon_{1}}\right)\right]\right\}, & \varepsilon_{1}<\bar{\varepsilon}<\varepsilon_{2} \\ 0, & \bar{\varepsilon} \geq \varepsilon_{2}\end{cases}
$$

The addition of equation (23) to the model accounts for the reduction in stiffness that is expected as damage accumulates in the aluminum matrix. We incorporated the constitutive law into the commercial finite element code ABAQUS [20] through a user-supplied subroutine UMAT, using a rate-tangent integration method by Peirce et al. [21].

\subsection{Determination of Material Parameters}

The most obvious method for the determination of material parameters would be standard uniaxial tensile tests on (unperforated) aluminum sheets. After material softening and subsequent localization of deformation, however, the mechanical behavior of the aluminum can only be indirectly inferred through further analysis. The anticipated failure mechanism in the perforated aluminum sheets is the localization of deformation between adjacent holes. For this reason, we decided to test sheets with a uniform square array of holes, and to infer the mechanical properties of the aluminum by finite element analyses of a corresponding unit cell. It is hoped that the 
parameters determined in this manner will be most appropriate for describing the softening behavior of the aluminum in the simulations using the selected representative unit cells.

More specifically, the tensile sample used to determine the material properties had a $10 \times 10$ array of $1.016 \mathrm{~mm}$ holes. The holes were uniformly spaced over an area with dimensions $28.5 \mathrm{~mm} \times$ $28.5 \mathrm{~mm}$ to give a hole area fraction of 10 percent. The simulation of the experiment was performed with a unit cell having a single hole in its center, and applying the appropriate periodic boundary conditions. Because of the additional symmetries of this particular periodic structure, only one-quarter of the cell had to be analyzed.

Two levels of mesh refinement were used in the simulations of the selected representative cells with twelve holes; the mesh of the simple unit cell used to ascertain material constants had approximately the same level of refinement as the coarser mesh used for the representative cells.

As in Becker and Smelser [10], the stress exponent was taken to be $m=100$, while the material parameters $\sigma_{y}, \sigma_{\max }, \varepsilon_{1}, \varepsilon_{2}$, and $n$ were adjusted to provide a fit to the experimental data. The experimental and numerical stress-strain curves are shown in Figure 2. With the level of discretization used in the calculations, the overall rate in which the stresses declined after maximum load was surprisingly insensitive to the value of the failure strain $\varepsilon_{2}$. Even when a value of $\varepsilon_{2}$ only slightly greater than $\varepsilon_{1}$ was used, the numerical simulations exhibited a significantly slower drop-off in stresses after maximum load than was observed in the experiments. One potential cause of this discrepancy is that fracture in the experiments occurs across only one row of holes, while the calculations implicitly assume that failure would occur across every row in the array of holes. The value of the failure strain was somewhat arbitrarily chosen to be $\varepsilon_{2}=0.66$, simply because relatively large values of $\varepsilon_{2}$ tended to increase the stable time step increment used in the computations. The failure mechanism exhibited in both the experiments and the simulations was strain localization directly across the ligaments between holes. The specific material parameters used to model the aluminum matrix are shown in Table I. 
Table I. Material Properties of aluminum 1100-H14.

\begin{tabular}{|l|l|}
\hline Properties & Value \\
\hline Initial Young's Modulus, $E_{0}$ & $72000 \mathrm{MPa}$ \\
\hline Poisson's Ratio, $v$ & 0.3 \\
\hline Yield Strength, $\sigma_{Y}$ & $9 \mathrm{MPa}$ \\
\hline Ultimate Strength, $\sigma_{\max }$ & $100 \quad \mathrm{MPa}$ \\
\hline Softening Strain, $\varepsilon_{1}$ & 0.36 \\
\hline Failure Strain, $\varepsilon_{2}$ & 100 \\
\hline Stress Exponent, $m$ & $0.00028 \quad s^{-1}$ \\
\hline Strain Rate, $\dot{\varepsilon}_{0}$ & 0.56 \\
\hline Strain Hardening Parameter, $n$ & \\
\hline
\end{tabular}

\section{Results}

The six complex patterns used in the experiments and the corresponding unit cells used in the finite element calculations are shown in Figure 3. In each case, the periodic hole patterns appear to have a similar spatial distribution as complex structures from which they were derived. The unit cells used for the finite element calculations are outlined in the lower left corner of the periodic structures. An example of the power spectral densities for the complex and periodic patterns is given in Figure 4.

Photographs of the tensile specimens, both before and after testing, are shown in Figure 5. During the early stages of loading, visible, localized deformation occurred around all of the holes. Thin ligaments between some of the holes subsequently necked and then failed. Upon further deformation, larger ligaments between holes necked primarily along the horizontal direction. The first major drop in load corresponded to rupture of a number of these ligaments, often extending to one edge of each of the specimens. 
Modeling localization and failure using the finite element method introduces a complicating factor into the analysis. Unfortunately, once localization of deformation occurs, the presence of the finite element mesh introduces an artificial length scale into the problem which in turn causes the solution to become mesh dependant. We therefore performed calculations on our representative unit cells with different element densities to ascertain the effects of mesh refinement on the numerical solutions. Each unit cell was therefore modeled with meshes of approximately 900 and 1800 plane stress, four node, quadrilateral elements.

Figures 6 compares the experimentally observed nominal stress-strain behavior with those predicted by the finite element calculations using the representative unit cells. Figure 6(a) shows the results from tensile experiments on perforated aluminum sheets. Figure $6(b)$ shows results for meshes with approximately 900 elements while Figure 6(c) corresponds to calculations with about 1800 elements. In almost every case, the figures show that the model calculations exhibit the same trends in strength and ductility as the six different hole patterns, although the calculations overestimate the ductility of the sheets by a significant margin in all cases. In one case, however (microstructure \#4), there is a significant discrepancy between the ductility predicted by the two different meshes.

Figure 7 displays contour plots of effective plastic strain just prior to failure in each of the representative unit cells modeled with approximately 1800 elements. In each case, the predicted failure path corresponds to the regions of maximum plastic strain. The effect of the periodic boundary conditions is evident, particularly for pattern $\# 1$, where the failure path meanders from the top to the bottom of the unit cell. The density of the finite element mesh used in the calculations is also clearly visible in the figures. Figure 8 compares contours of plastic strain for the microstructure $\# 4$, the case where the different mesh densities gave significantly different predictions of sheet ductility. In this particular case, competing failure paths developed in the analysis with the finer mesh, which in turn resulted in a significant increase in the predicted ductility when compared with the coarse mesh.

In order to more clearly see the trends exhibited in the experiments and by the corresponding models, we also compared values of the relative ultimate strengths in Figure 9. In Figure 9, the 
experimental strengths have all been normalized by the measured ultimate strength of hole pattern \#1. In contrast, the predicted strengths have been normalized to provide a "best fit" to the trends measured experimentally. From this perspective, the model also provides reasonably good estimates of the trends in ultimate strength exhibited by the perforated sheets.

\section{Discussion}

In this investigation, idealized two-phase microstructures were constructed by perforating sheets of aluminum with random patterns of holes. They were then subjected to uniaxial tension tests so that nominal stress-strain relationships could be obtained. Each of the complex hole patterns were modeled using representative unit cells with a statistically similar distribution of holes. The unit cells were obtained by comparing the power spectral densities of the image of the original random hole pattern with that of an idealized periodic pattern [13]. The resultant boundary value problem was solved by use of finite elements. The experimental results show the strong effect that hole distribution can have in thin sheets, which is also expected in other composite materials. The predicted nominal stress-strain curves follow the trends in ductility variations between the various microstructures, although an over-prediction of ductility is consistently observed.

There are a number of possible explanations as to why the model overestimated the observed ductility in every case. First, the calculations prescribe periodic boundary conditions on the representative unit cell, so that the effect of free edges on the behavior of the actual tensile specimens is not accounted for in the analysis. The presence of edges in these finite-width specimens may allow for premature failure of ligaments near the edges so that the observed ductility is lower in comparison to an infinitely large specimen. Second, the material model and parameters used in the calculations are highly simplified and may not accurately represent the behavior of the aluminum. A third possible reason is that failure will occur along the weakest of all possible failure paths in the test specimen. The chosen periodic structure, however, is only in some sense typical of the actual distribution of holes in the aluminum test specimens. The unit cell used in the finite element calculations is therefore unlikely to have a potential failure path that is as weak as the path observed experimentally. The final and most likely reason for the discrepancies, however, is simply that failure in the model will occur, by definition, across every unit cell that 
make up the periodic hole pattern. The fact that the model has, in essence, multiple failure paths leads to an over prediction of the ductility of the perforated sheets.

This idea can also be used to explain the differences in the ductility predicted by the different levels of mesh refinement for microstructure \#4. In the analysis with the finer mesh, an additional potential fracture path developed that significantly increased the predicted ductility. This result suggests that ductility predictions may be quite sensitive to small variations in unit cell geometry and material constitutive behavior, as well as the level of mesh refinement. In contrast, we expect that predictions of ultimate strength (and deformation behavior prior to localization) would be relatively insensitive to small variations in model parameters.

It is worth noting some other important limitations in the methods described in this paper. One obvious limitation is that the method is restricted to two-dimensional microstructures, while composites with short fiber or particle reinforcements are clearly three-dimensional in nature. Even for three-dimensional microstructures, however, it may be possible to adequately model the composite with a two-dimensional, generalized plain strain calculations. A more significant limitation in our view, however, is that the many composite microstructures (see, for example, Park et al. [22]) have regions with large clusters of particles, and conversely, regions with little or no reinforcement. Such a microstructure would require a unit cell with much larger numbers of reinforcements than those used in the present study, which could in turn render the associated finite element calculations overly complex and computationally intractable.

The numerical methods used in this study could also be improved upon. For example, for composites that have particles of various size, morphology, and orientation, it is presently unclear as to how representative reinforcements should be selected. Moreover, there are very likely better ways to compare the spectral densities of the complex and periodic microstructures, and better ways to find the minimum of the residual function $\chi^{2}$ (equation (12)) to ensure that the absolute minimum (and therefore the most representative unit cell) is found. Perhaps techniques used in image analysis or pattern recognition could be used to improve upon or even supplant the numerical methods used here. 
Despite the limitations outlined above, however, we believe that the central ideas of this study have been validated. With our experiments, we have shown that the spatial distribution of a second phase can significantly affect the mechanical behavior, particularly regarding the evolution of damage and eventual failure of the material. Using representative unit cells that have a statistically similar distribution of holes to that of the perforated aluminum sheets, we have successfully predicted the effects that the hole distribution had on the behavior of the sheets.

\section{Acknowledgements}

The authors are pleased to acknowledgement financial support provided by the Air Force Office of Scientific Research, contract number F49620-96-1-0198.

\section{References}

1. Lewandowski, J.J., Liu, C. and Hunt, W. H., Mater. Sci. Engrg., 1989, A107, 241.

2. Bao, G., Hutchinson, J. W. and McMeeking, R. M. , Acta Metall. Mater., 1991, 39, 1871.

3. Christman, T., Needleman, A. and Suresh, S. , Acta Metall., 1989, 37, 3029.

4. Koplik, J. and Needleman, A., International Journal of Solids \& Structures., 1988, v24 n8, 835

5. Needleman, A., J. appl. Mech., 1987, 54, 525.

6. Finot, M., Shen, Y-L, Needleman, A., and Suresh, S., Metall. Mater. Trans., 1994, A25, 2403.

7. Llorca, J., Needleman, A. and Suresh, S., Acta Metall. Mater., 1991, 39, 2317.

8. Povirk, G. L., Stout, M. G., Bourke, M., Goldstone, J., Lawson, A. C., Lovato, M., MacEwen, S. R., Nutt, S. R. and Needleman, A., Acta Metall. Mater., 1992, 40, 2391.

9. Magnuson, P. E., Dubensky, E. M. and Koss, D. A., Acta Metall., 1988, 36, 1503.

10. Becker, R. and Smelser, R. E., J. Mech. Phys. Solids, 1994, 42, 773.

11. Ostoja-Starzewski, M., Sheng, P. Y. and Jasiuk, I., J. Eng. Mater. Tech., 1994, 116, 384.

12. Moorthy, S., and Ghosh, S., Comput. Methods Appl. Mech. Engrg., 1998, 151, 377.

13. Povirk, G. L., Acta Metall. Mater., 1995, 43, 3199.

14. Baker, H. et al., eds., Metals Handbook, vol. 2 (Metals Park, OH: American Society for Metals), 1979, 29.

15. LabVIEW ${ }^{\mathrm{TM}}$ version 4.0 (National Instruments Corporation 1996, www.ni.com)

16. Press, W.H., Teukolsky, S.A., Vetterling, W.T. and Flannery, B.P. Numerical Recipes in Fortran (Cambridge, U.K.: Cambridge University Press), 1992. 
17. Parzen, E., Stochastic Processes (Holden-Day, San Francisco, California), 1962, 110.

18. Hirsch, P. B., Howie, A., Nicholson, R. B., Pashley, D. W. and Whelan, M. J., Electron Microscopy of Thin Crystals ( $2^{\text {nd }}$ Edition, Kreiger, Malabar, Florida), 1977.

19. HyperMesh ${ }^{\mathrm{TM}}$ version 2.1(Altair Computing, Inc. 1997, www.altair.com)

20. ABAQUS ${ }^{\mathrm{TM}}$ version 5.7 (Hibbitt, Karlsson \& Sorensen, Inc. 1997, www.hks.com)

21. Peirce, D., Shih, C. F. and Needleman, A., Comp. Struct., 1984, 18, 875.

22. Park, J., Lenshek, D. X., and Povirk, G. L., Acta Mater., 1997, 45, 1351. 


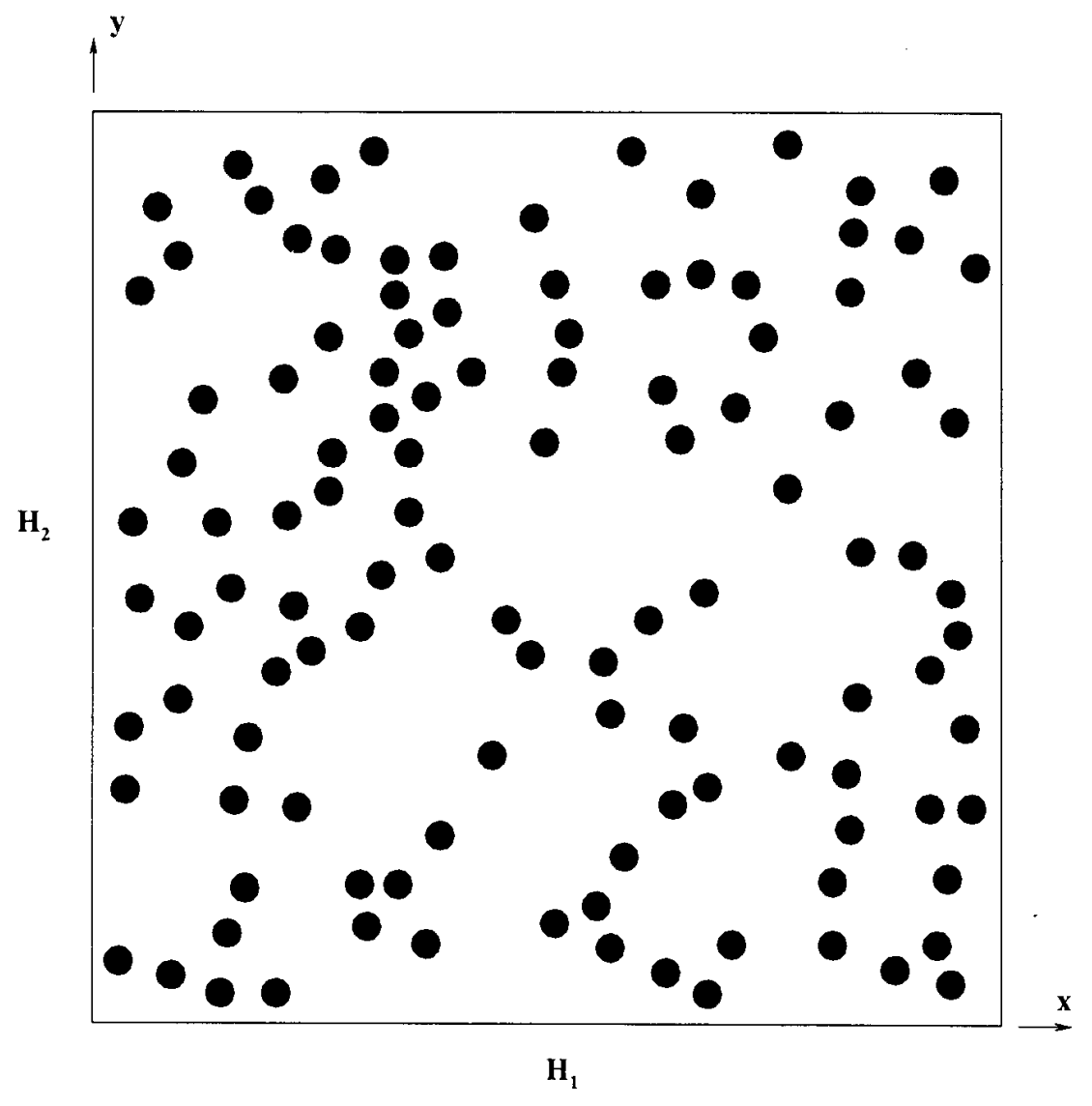

Figure 1. Typical hole pattern used for the perforated aluminum sheets. 


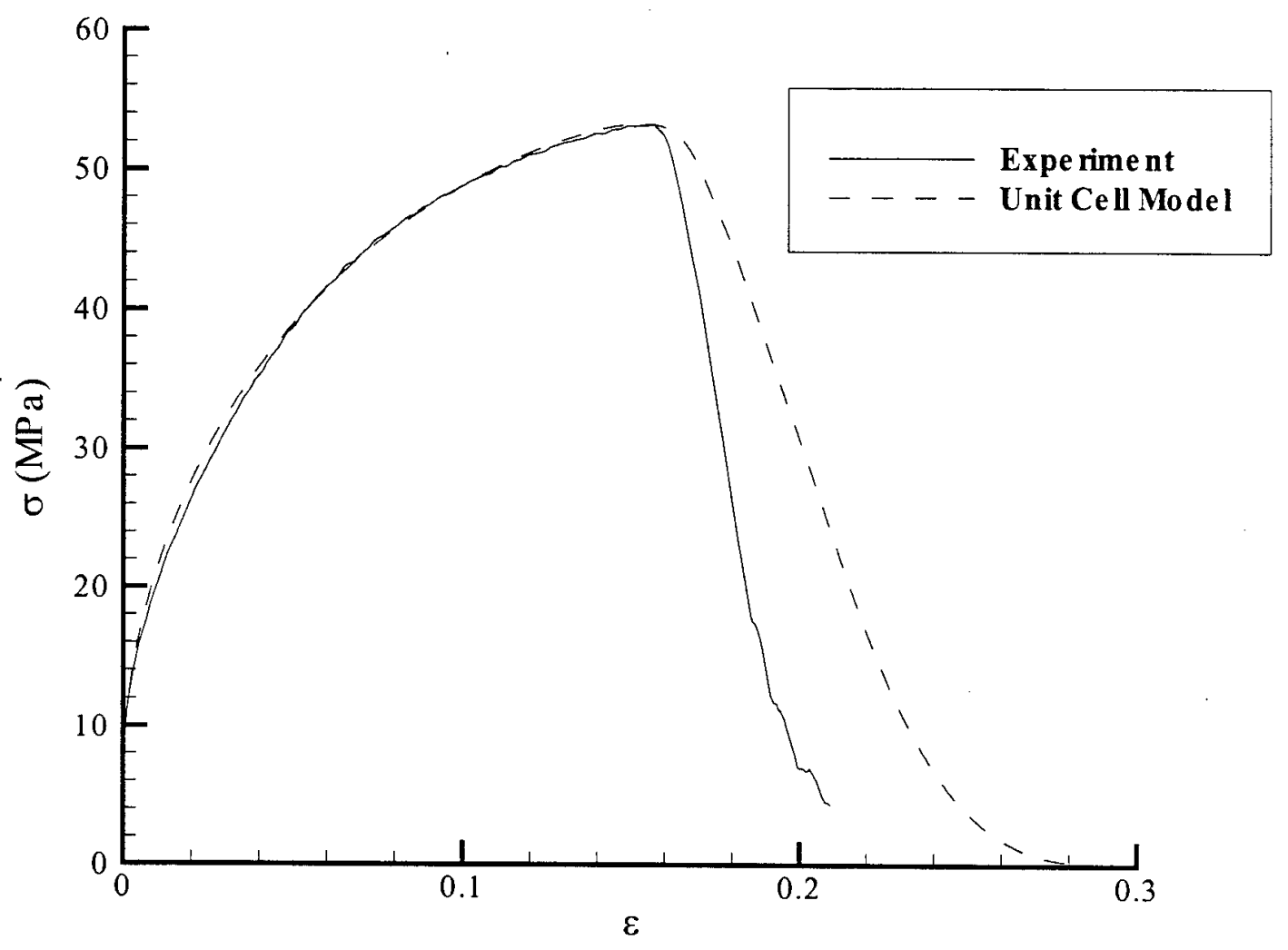

Figure 2. The solid line represents the nominal stress vs. strain curve for a perforated aluminum sheet with a simple cubic pattern of holes and a volume fraction of ten percent. The dashed line is the corresponding predicted response of a simple unit cell using the material properties listed in Table I. 

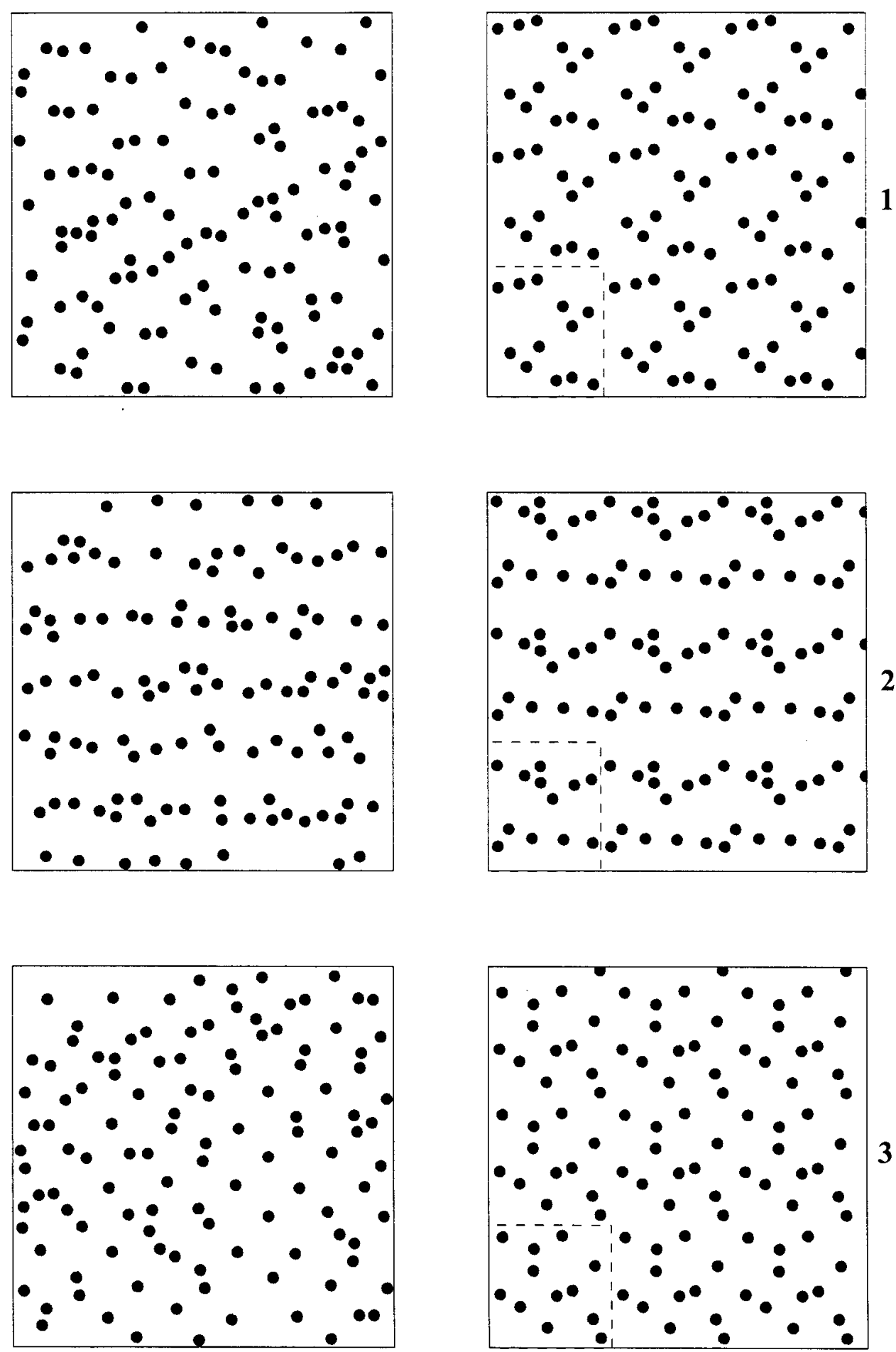

Figure 3. Diagrams of the 6 microstructures used in perforated aluminum sheets (left) and their corresponding periodic structures (right). The unit cells used in finite element calculations are indicated by dashed rectangles. 
Perforated Sheet Microstructure

Periodic Structure
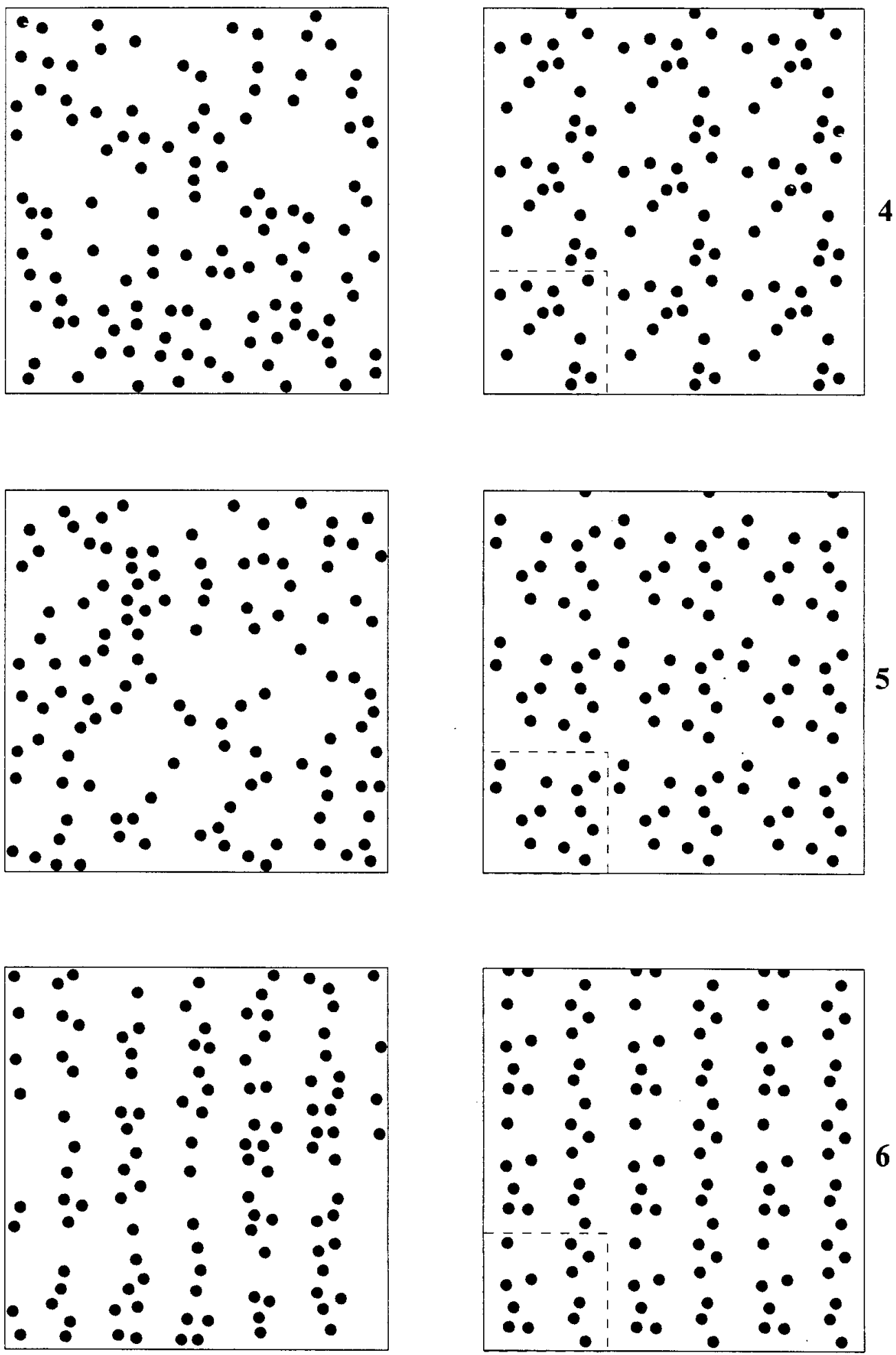

Figure 3. (continued) 

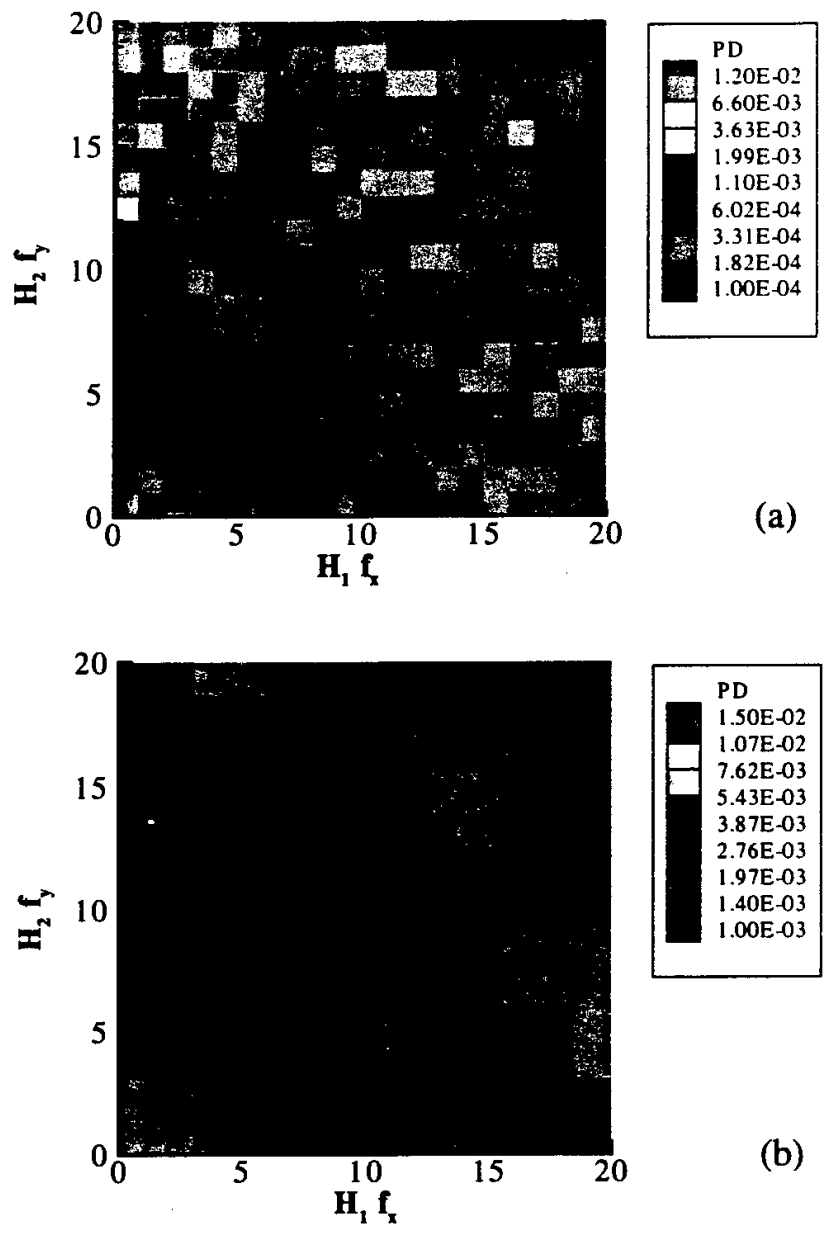

(b)
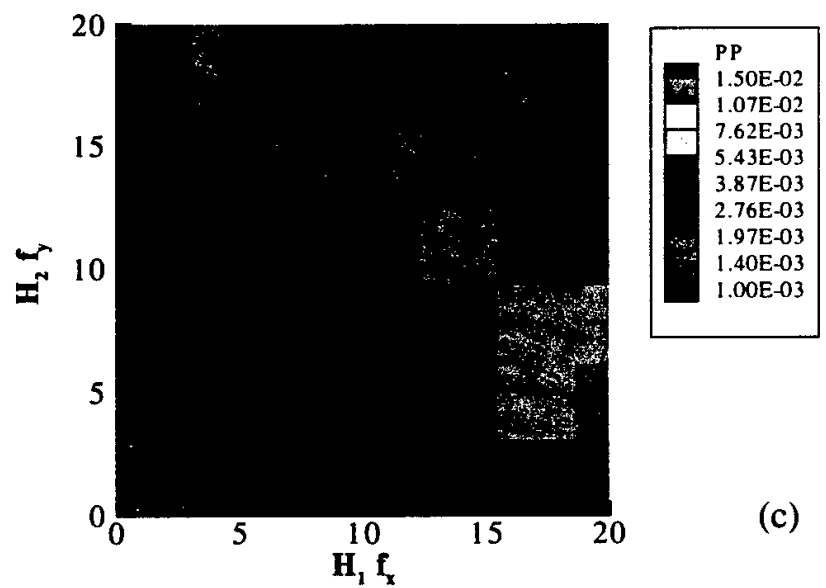

(c)

Figure 4. Power spectral densities of (a) the original hole pattern; (b) the original hole pattern after "rebinning"; and (c) the corresponding periodic structure. 
Before tensile experiment
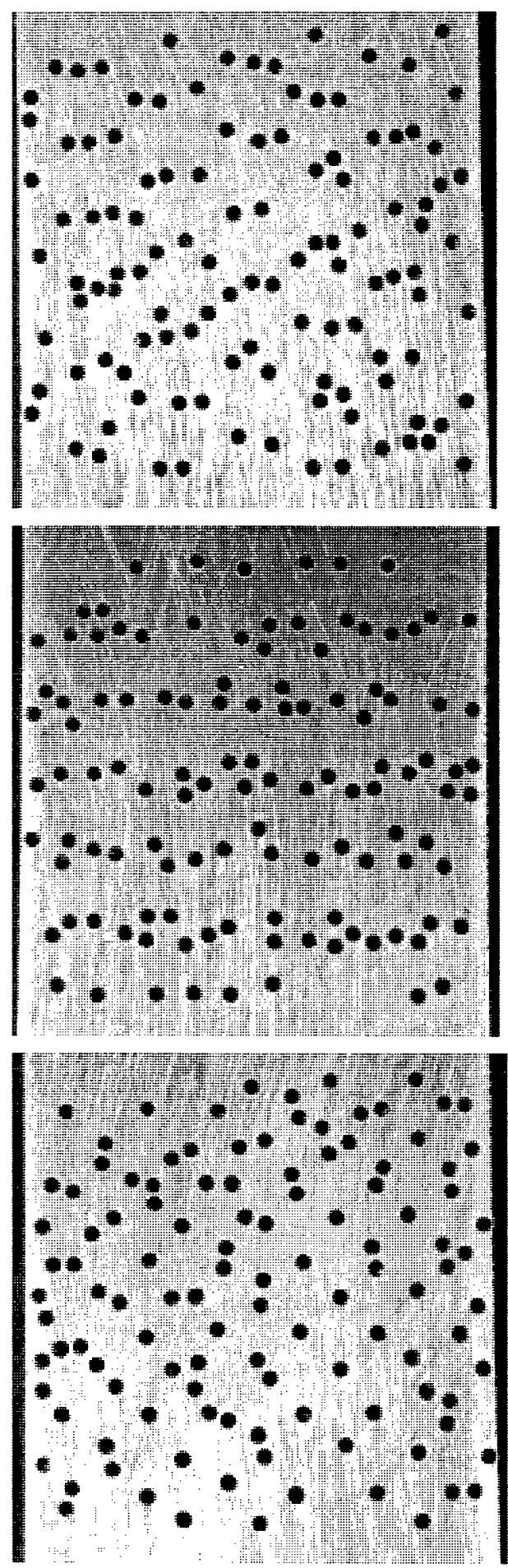

After tensile experiment
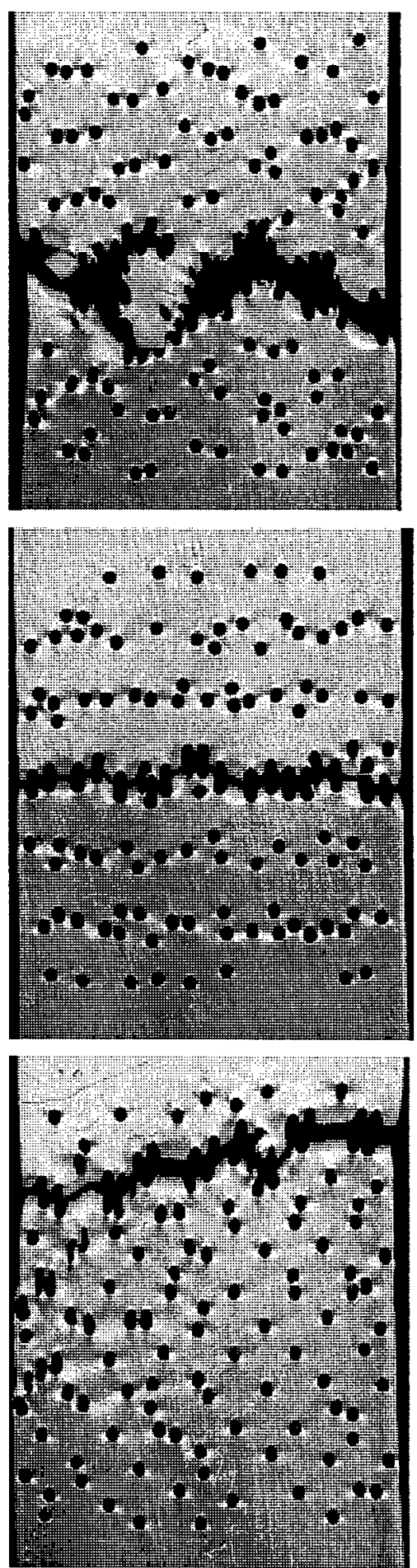

Figure 5. Photographs of the perforated aluminum sheets before and after the uniaxial tensile experiments. 
Before tensile experiment
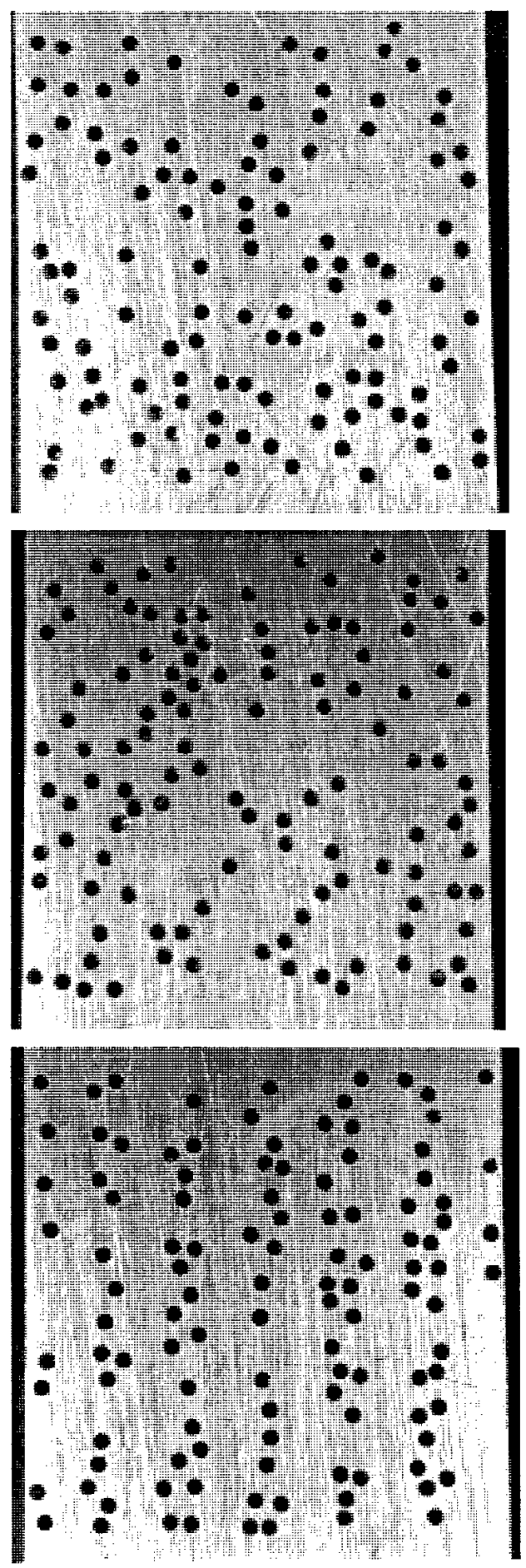

After tensile experiment
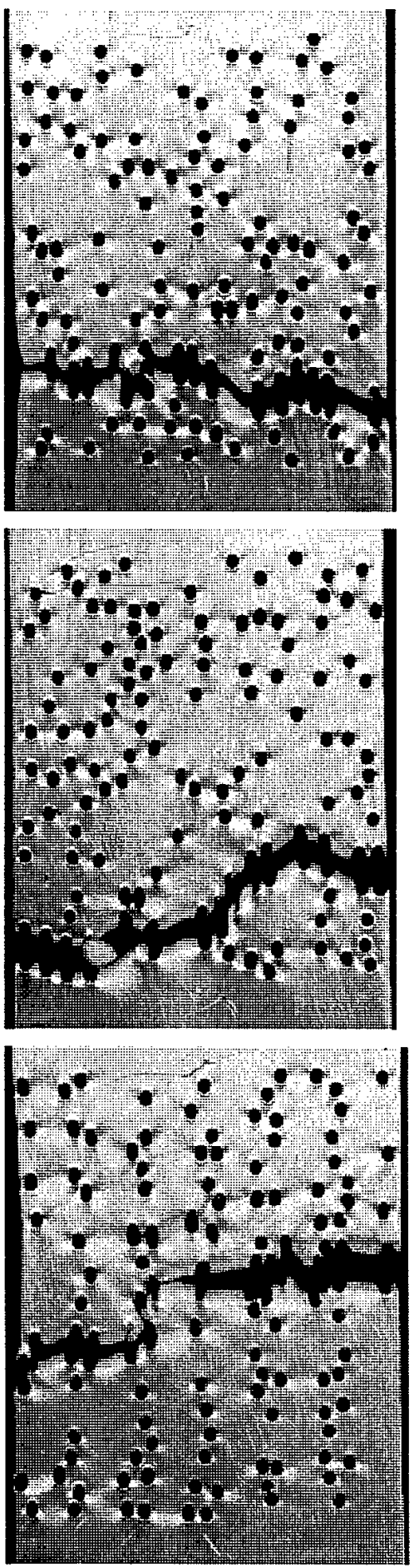

Figure 5. (continued) 


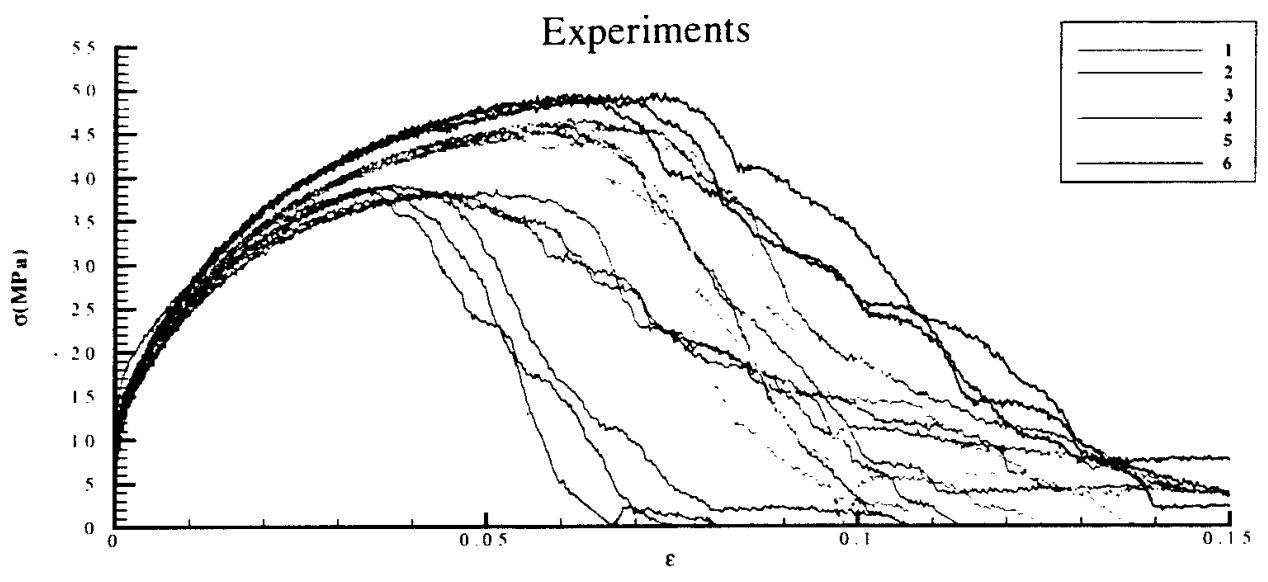

(a)

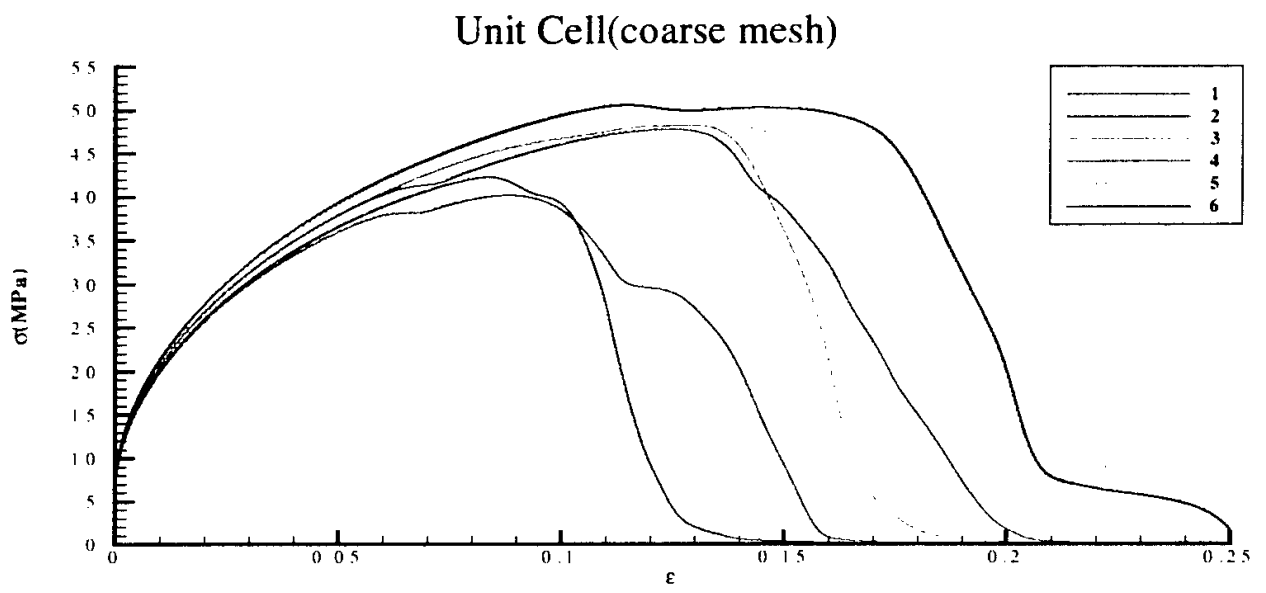

(b)

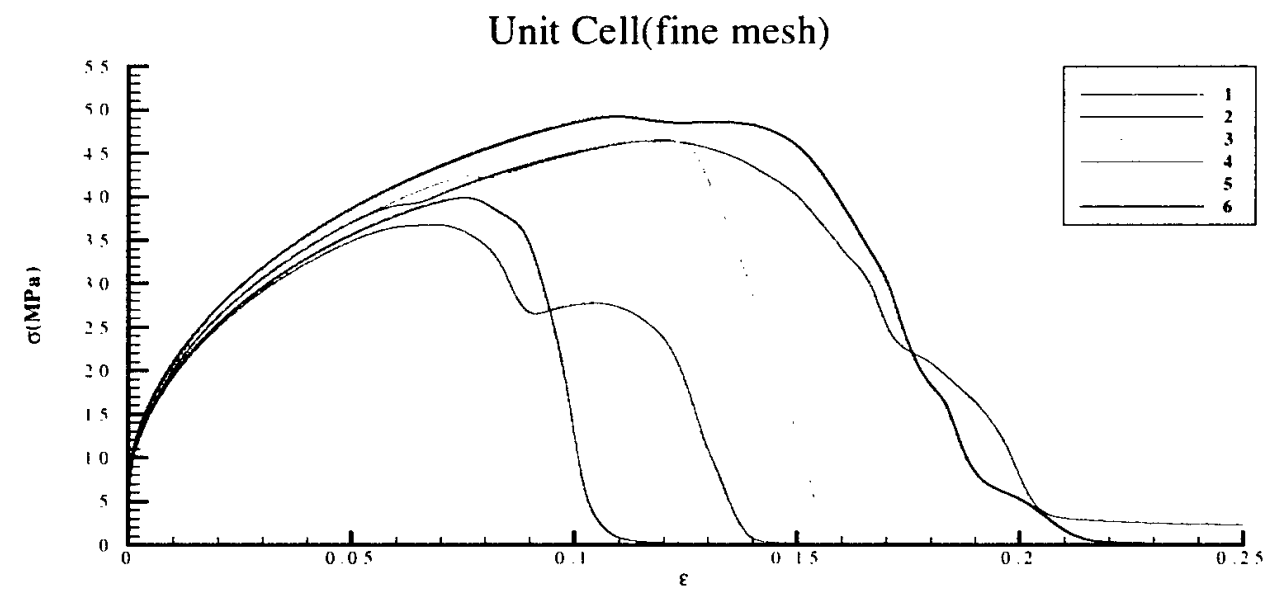

(c)

Figure 6. Stress vs. strain behavior of (a) the perforated aluminum sheets; (b) the corresponding unit cell calculations with approximately 900 elements; (c) unit cell calculations with approximately 1800 elements. Each color represents a different hole pattern; each hole pattern was tested three times experimentally. 


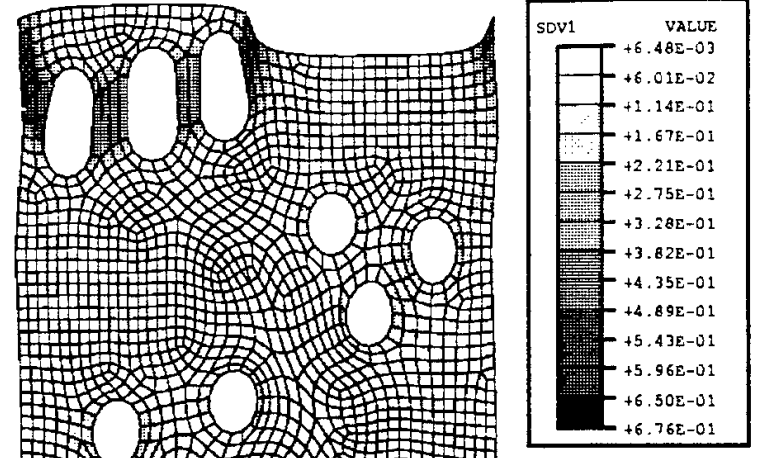

1
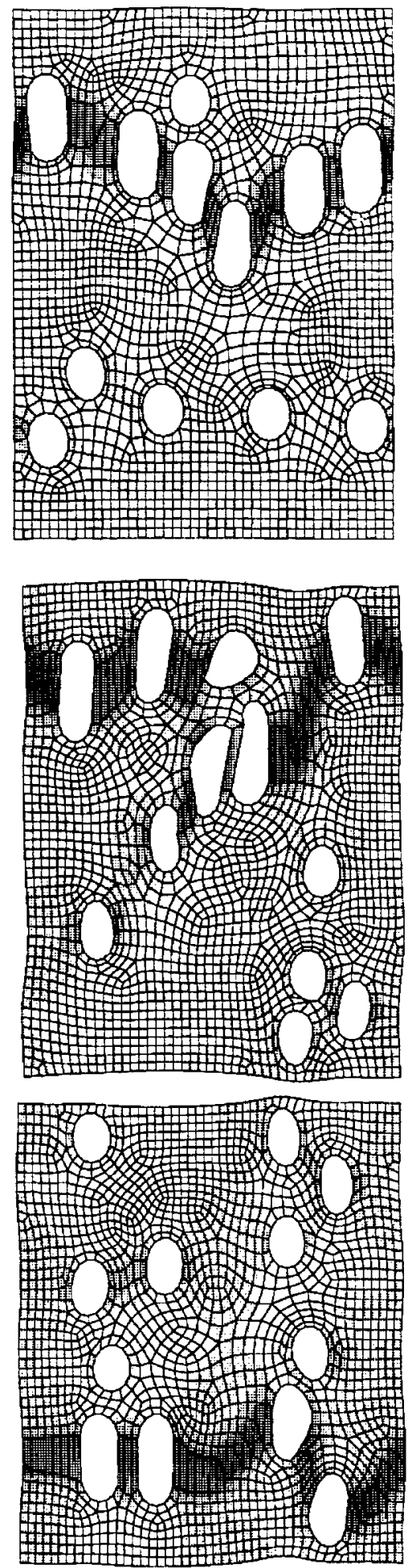

Figure 7. Contour plots of accumulated effective plastic strain for representative unit cells. Regions of dark shading indicate elements that have completely failed. 

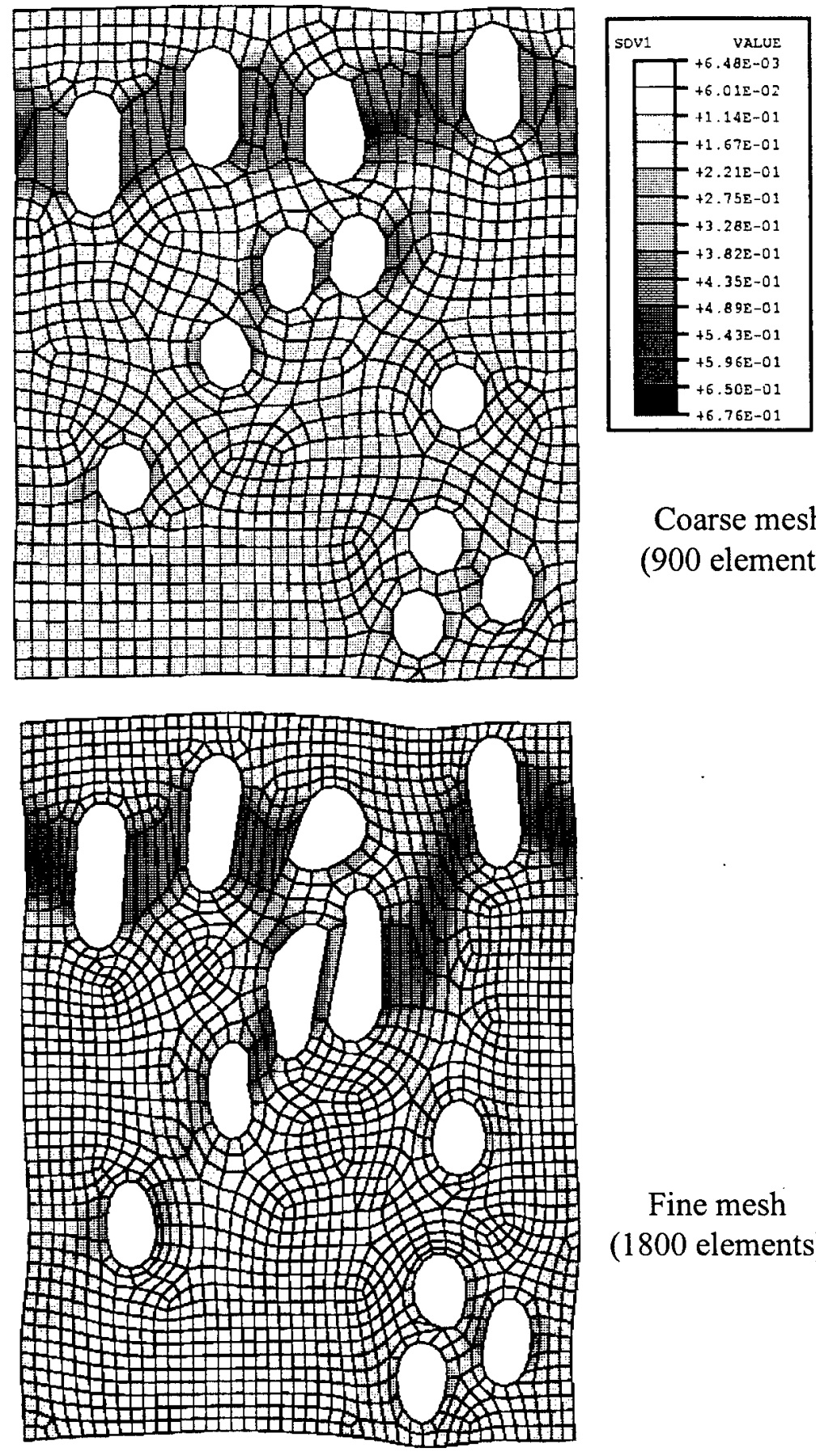

Coarse mesh

(900 elements)

Fine mesh

(1800 elements)

Figure 8. Contour plots of effective plastic strain of unit cell \#4 with coarse and fine mesh. For this particular unit cell, competing failure paths develop with the fine mesh, which in turn causes an increase in predicted ductility. 


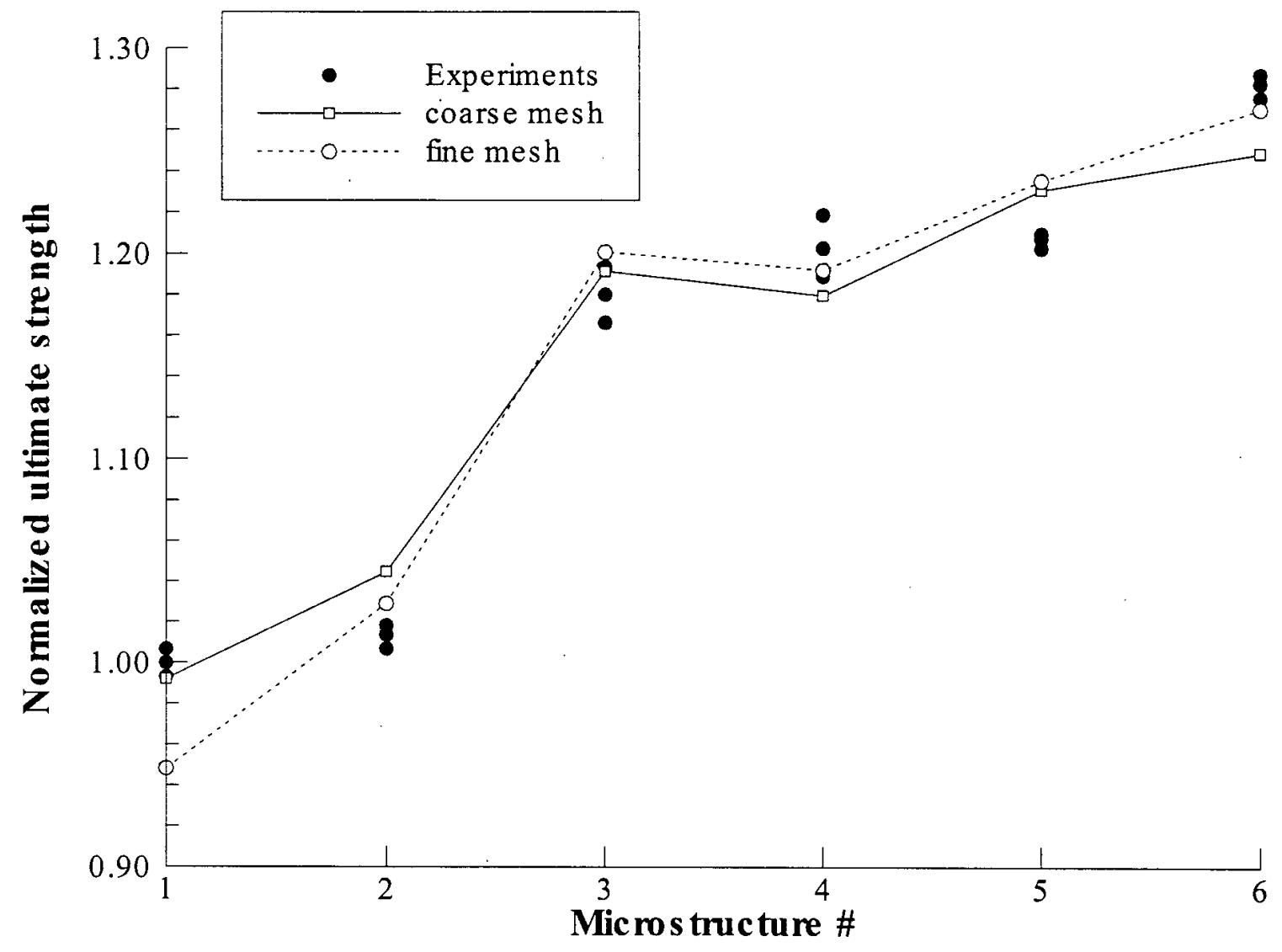

Figure 9. Normalized ultimate strengths of the perforated aluminum sheets compared to the strengths predicted by the unit cell calculations. 


\section{REPORT DOCUMENTATION PAGE}

Public reporting burden for this collection of information is estimated to average 1 hour per response, including gathering and maintaining the data needed, and completing and reviewing the collection of information. Send $c$

collection of information, including suggestions for reducing this burden. to Washington Headquarters Services,

collection of information, including suggestions for reducing this burden. to Washington Headquarters Services,

AFRL-SR-BL-TR-00-

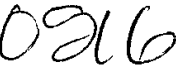

\begin{tabular}{|l|l|l|l} 
1. AGENCY USE ONLY (Leave & 2. REPORT DATE & 3. REPORT TYPE mivu umico uUv cMcu
\end{tabular} Blank) $9 / 12 / 00$ Final, 5/1/96 - 4/30/99

4. TITLE AND SUBTITLE

Modeling and Process Control of Composite Microstructures

5. FUNDING NUMBERS

F49960-96-1-0198

6. AUTHORS

Gary L. Povirk

7. PERFORMING ORGANIZATION NAME(S) AND ADDRESS(ES)

Yale University

P.O. Box 208337

New Haven, CT 06520-8337

9. SPONSORING / MONITORING AGENCY NAME(S) AND ADDRESS(ES)

Air Force Office of Scientific Research

10. SPONSORING / MONITORING AGENCY

110 Duncan Avenue, Room B115

Bolling AFB, DC 20332-8050

8. PERFORMING ORGANIZATION REPORT NUMBER

11. SUPPLEMENTARY NOTES

12a. DISTRIBUTION / AVAILABILITY STATEMENT

12b. DISTRIBUTION CODE

UL

13. ABSTRACT (Maximum 200 words)

See final report

14. SUBJECT TERMS

15. NUMBER OF PAGES

16. PRICE CODE

17. SECURITY CLASSIFICATION
18. SECURITY CLASSIFICATION OF REPORT

Unclassified

NSN 7540-01-280-5500
Undassified

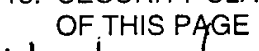

19. SECURITY CLASSIFICATION Un ABSTRAGTified

\begin{tabular}{|l|l|}
\hline 4. & TITLE AND SUBTITLE \\
Modeling and Process Control of Composite Microstructures & FUNDING NUMBERS \\
F49960-96-1-0198
\end{tabular}

20. LIMTTATIONOF

ABSTRACT

$$
\text { UL }
$$

Standard Form 298 (Rev. 2-89) Prescribed by ANSI Std. Z39- 1 298-102 


\title{
MODELING THE EFFECTS OF HOLE DISTRIBUTION IN PERFORATED ALUMINUM SHEETS I : REPRESENTATIVE UNIT CELLS
}

\author{
G. F. Raiser, S. Jia, and G. L. Povirk \\ Department of Mechanical Engineering \\ Yale University \\ New Haven, Connecticut 06520 USA
}

\begin{abstract}
A method is presented that incorporates microstructural information into a model of the mechanical behavior of two-phase composite materials. The approach is to determine periodic microstructures that are statistically similar to the actual microstructure of the material under consideration. The utility of this method is that computationally tractable finite element simulations can then be carried out on representative unit cells that are directly obtained from microstructural observations. To illustrate this method, mechanical tests are performed on perforated aluminum sheets with various microstructures, and the results are compared to finite element simulations of selected representative unit cells. The simulations agree with the trends observed in the experiments, including measurements of the overall strength and ductility of the sheets. Advantages and limitations of the approach used here are discussed.
\end{abstract}




\section{Introduction}

Materials with two distinct phases represent a broad class of materials, including dispersionstrengthened alloys, mechanically-alloyed materials, spheroidized steels, and metal- and ceramicmatrix composites. Incomplete densification during processing, creep cavitation, or void nucleation during ductile fracture can cause even nominally homogeneous materials to contain a significant fraction of voids. These voids can also be thought of as secondary phase. The microstructure of a two-phase material can in turn have a profound effect on the mechanical properties of the material, particularly on the initiation and evolution of damage. For example, research by Lewandowski et al. [1] on aluminum-silicon carbide composites has shown that fracture usually initiates at large particles, inclusions, and regions of clustered silicon carbide particles. They also observed that damage accumulation ahead of a macroscopic crack tends to occur in regions where reinforcements are clustered together.

Many investigators have studied two-phase materials by idealizing the composite microstructure as having a simple periodic structure, with one or two reinforcing particles (or voids) within each unit cell. With this assumption, estimates of the macroscopic response of the material can be obtained by solving an appropriate boundary value problem formulated on a representative unit cell. This approach has been used to estimate the flow behavior of metal-matrix composites [2,3] and of porous metals [4]. Moreover, simple unit cell models have be used to study the damage mechanisms in composite materials, such as interfacial debonding [5], particle cracking [6], and ductile failure in the matrix [7]. While these analyses have provided important insights into deformation and damage initiation in these materials, they also possess significant limitations as well. One important limitation is that the predicted behavior can be strongly affected by the choice of assumed unit cell parameters [2,8]. Furthermore, a simple unit cell with one reinforcement (or void) cannot provide a realistic portrayal of damage evolution in a composite material. For example, experiments on aluminum sheets containing randomly distributed perforations showed the pronounced effects of hole distribution on resultant mechanical behavior [9]. Corresponding finite element calculations by Becker and Smelser [10] showed that a simple unit cell with one hole could not adequately predict the ductility of the sheets. 
More recently, investigators have begun to account for the effects of microstructure on the mechanical properties of two-phase materials. A central problem that develops, however, is the computational expense associated with incorporating large amounts of microstructural information into the model. To make the problems more tractable, researchers have used different procedures to simplify the problem. For example, Ostoja-Starzewski et al. [11] developed a finite difference lattice model in which damage evolution is simulated by sequentially removing bonds in the model if the lattice strain exceeds a critical value. While this method is quite useful in examining the effects of material constants on the qualitative behavior of composite materials, more detailed descriptions of material behavior are required to model real composite materials. Ghosh et al. [12] has developed a model in which a composite material is divided into Voronoi cells, with each cell containing one reinforcement at most. For each Voronoi cell, a stress hybrid method is used where an equilibrated stress field (derived from an Airy stress function) is assumed within the cell and displacements are interpolated on the boundary of the cell. While the method is complex, it appears to have great potential in modeling deformation and damage within composite materials.

The intent of this work is to develop alternative tools for incorporating microstructural information into models of two-phase materials. The approach considered here is to determine a periodic microstructure that is statistically similar to the actual microstructure under consideration. With a periodic microstructure so determined, a numerical analysis can be performed on a single unit cell of the periodic structure, with an associated reduction in computational cost. To test the procedures, idealized composites are constructed by perforating aluminum sheets with various complex patterns of holes and subjecting the specimens to standard uniaxial tensile tests. After corresponding periodic microstructures have been determined, finite element simulations of uniaxial tensile tests are performed on representative unit cells and the results are compared to experimental results. This study is a continuation of an investigation by Povirk [13], who compared the elastic behavior of complex microstructures to that of selected representative unit cells.

\section{Procedures}

The general procedures used in this study are outlined as follows. Six complex patterns of circles were generated and used as templates to obtain aluminum sheets with various hole distributions. 
The mechanical behavior of the perforated sheets was then determined experimentally by uniaxial tensile testing. Numerical models of the six complex microstructures were developed by first obtaining corresponding unit cells that have similar spatial distributions of holes; the methods used in the determination of the unit cell parameters will be discussed subsequently. Finite element meshes were then generated for each of the six unit cells, and appropriate periodic boundary conditions were implemented. The flow behavior of the aluminum matrix was characterized by isotropic, rate-dependent $J_{2}$ flow theory, with initial strain-hardening followed by eventual strainsoftening. The behavior predicted by the unit cell models were then compared to the corresponding experimental results.

\subsection{Generation of Hole Patterns}

The region in which the hole patterns were generated were assumed to occupy a rectangular region of dimensions $\mathrm{H}_{1} \times \mathrm{H}_{2}$ (see Figure 1). Each pattern was arbitrarily assumed to contain 115 holes, with the hole diameter chosen such that the area fraction was 10 percent. The initial step was to discretize this region into an $N_{1} \times N_{2}$ array of points, given by

$$
x_{k_{\mathrm{s}}}=\frac{H_{1}}{N_{1}} k_{1}, \quad k_{1}=0, \ldots, N_{1} \quad ; \quad y_{k_{1}}=\frac{H_{2}}{N_{2}} k_{2}, \quad k_{2}=0, \ldots, N_{2}
$$

The hole patterns were generated by assigning, at each of the discrete points defined by equation (1), a number $p$ of the form

$$
\begin{aligned}
p & =r+\varepsilon_{1} \cos \left(\frac{12 \pi x}{H_{1}}\right)+\varepsilon_{2} \cos \left(\frac{12 \pi y}{H_{2}}\right) \\
& +\frac{\varepsilon_{3}}{2}\left[\sin \left(\frac{12 \pi x}{H_{1}}-\frac{\pi}{2}\right) \sin \left(\frac{12 \pi y}{H_{2}}-\frac{\pi}{2}\right)+1\right] \\
& +\frac{\varepsilon_{4}}{2}\left[\sin \left(\frac{12 \pi x}{H_{1}}-\frac{\pi}{2}\right) \sin \left(\frac{6 \pi y}{H_{2}}-\frac{\pi}{2}\right)+1\right] \\
& +\frac{\varepsilon_{5}}{2}\left[\sin \left(\frac{6 \pi x}{H_{1}}-\frac{\pi}{2}\right) \sin \left(\frac{12 \pi y}{H_{2}}-\frac{\pi}{2}\right)+1\right]
\end{aligned}
$$

where $r$ represents a random number ranging from 0 to 1 , and the variables $\varepsilon_{i}$ are input parameters that were used to exert a measure of control over the generated patterns of holes. Circles were 
placed starting at the point with the maximum value of $p$ and sequentially thereafter at points with descending values of $p$. Any new circle location that overlapped or touched an existing circle was rejected. Six different microstructures were generated by selecting an initial "seed" for the random number generator and choosing different values of the parameters $\varepsilon_{i}$. Templates for the aluminum sheets were generated based on the calculated hole coordinates.

\subsection{Experimental Procedure}

Specimens were machined from 1100-H14 aluminum sheets. Each sample has a thickness of 0.81 $\mathrm{mm}$, a width of $30.5 \mathrm{~mm}$ and a gauge length of $30.5 \mathrm{~mm}$. The hole patterns were printed out on a laser printer and bonded to the aluminum tensile samples. With the template attached to the specimens, holes with a diameter of $1.016 \mathrm{~mm}$ were drilled in the appropriate locations. For each of the six patterns that were generated, three identical samples were prepared. After drilling, any excessive metal extending out of the holes was sanded off, and the specimens were subjected to annealing at $400^{\circ} \mathrm{C}$ for $60 \mathrm{~s}$. (O-temper [14]).

Tensile tests were conducted on an MTS servo-hydraulic testing machine configured with a 458.20 MicroConsole using a TestLink interface. The MicroConsole was equipped with a DC controller for control and data acquisition of the loads and an AC controller for control and data acquisition of the displacement. A MTS 632.11B-20 extensometer with an attachment kit for flat sample applied was used to measure the strain of the sample. The default distance between the two knife edges of the extensometer was $25.4 \mathrm{~mm}$ but was modified to be $30.5 \mathrm{~mm}$ to match the gauge length of the sample. Another DC controller on the Microconsole was used for control and data acquisition of the strain data from the extensometer.

One difficulty involved in the experiments was that as failure progressed, significant rotation of the specimen would often occur. This is an important problem, since the model of sheet deformation does not account for this effect. To limit this effect, we first applied a small tensile load to align the sample. Once the specimen is aligned, we then tightened two adjustable "stops" on each side of specimen to limit the rotation of the specimen during testing. The extensometer was then attached to the sample, and a constant velocity of $0.04 \mathrm{~mm} / \mathrm{s}$ was prescribed to the lower grip. The load, displacement and strain (actually distance between two knife edges of the extensometer) data was 
output to a computer through a LAB-PC data acquisition expansion board. A LabVIEW [15] program was created to process these data and output the engineering stress and strain of the sample. Each sample was pulled to complete failure.

\subsection{Selection of Unit Cell Parameters}

The first step in the selection of unit cell parameters is to represent a complex pattern of holes by a discrete indicator function

$$
g^{c}\left(x_{k_{1}}, y_{k_{2}}\right)= \begin{cases}0 & \text { for phase } 1 \\ 1 & \text { for phase } 2\end{cases}
$$

where phase 1 and phase 2 represents the aluminum matrix and the holes, respectively. The corresponding unit cell that will be used for the finite element calculations is described by a similar indicator function.

The power spectral density of the complex patterns is obtained at discrete frequencies by first calculating the two-dimensional, discrete, fast Fourier transform of the indicator function, $G^{c}\left(f_{x}^{n_{1}}, f_{y}^{n_{2}}\right)$

$$
G^{c}\left(f_{x}^{n_{1}}, f_{y}^{n_{2}}\right)=\sum_{k_{2}=0}^{N_{2}-1} \sum_{k_{1}=0}^{N_{1}-1} \exp \left(2 \pi i x_{k_{1}} f_{x}^{n_{1}}\right) \exp \left(2 \pi i y_{k_{2}} f_{y}^{n_{2}}\right) g^{c}\left(x_{k_{1}}, y_{k_{2}}\right)
$$

at frequencies given by

$$
f_{x}^{n_{1}}=\frac{n_{1}}{H_{1}}, \quad n_{1}=-\frac{N_{1}}{2}, \ldots, \frac{N_{1}}{2} \quad f_{y}^{n_{2}}=\frac{n_{2}}{H_{2}}, \quad n_{2}=-\frac{N_{2}}{2}, \ldots, \frac{N_{2}}{2}
$$

Because the indicator function is real, its Fourier transform is independent of the signs of the frequencies $f_{x}^{n_{1}}$ and $f_{y}^{n_{2}}$. The one-sided power spectral density of the complex pattern, $P^{c}\left(f_{x}^{n_{1}}, f_{y}^{n_{2}}\right)$, can then be written as [16]

$$
P^{c}\left(f_{x}^{n_{1}}, f_{y}^{n_{2}}\right)= \begin{cases}\left|G^{c}\left(f_{x}^{n_{1}}, f_{y}^{n_{2}}\right)\right|^{2} & \text { for } n_{1}, n_{2}=0 \\ 2\left|G^{c}\left(f_{x}^{n_{1}}, f_{y}^{n_{2}}\right)\right|^{2} & \text { for } n_{1}>0, n_{2}=0, \text { or } n_{1}=0, n_{2}>0 \\ 4\left|G^{c}\left(f_{x}^{n_{1}}, f_{y}^{n_{2}}\right)\right|^{2} & \text { for } n_{1}, n_{2}>0\end{cases}
$$


The use of the fast Fourier transform requires that both $N_{1}$ and $N_{2}$ be powers of two. In the following discussions, frequencies with indices of $n_{1}$ and $n_{2}$ will refer to those associated with the complex hole patterns.

The next step is to choose the desired number of holes for the unit cell, an initial set of dimensions $h_{1}$ and $h_{2}$ for the cell, and an initial set of hole positions. The dimensions for the cell were chosen to yield the same area fraction of holes as in the complex patterns. The indicator function for the unit cell is then written as

$$
g^{p}(x, y)=\sum_{\beta=1}^{n_{p}} g_{0}\left(x-\phi_{x}^{\beta}, y-\phi_{y}^{\beta}\right)
$$

where $g_{0}(x, y)$ describes the shape of the holes, $\phi_{x}^{\beta}$ and $\phi_{y}^{\beta}$ are the coordinates of hole $\beta$ and $n_{p}$ is the number of holes in each unit cell. For this study, each unit cell was assumed to contain twelve holes. The unit cell is discretized by an $M_{1} \times M_{2}$ array of points so that the fast Fourier transform of the periodic structure then takes the form

$$
G^{p}\left(f_{x}^{m_{1}}, f_{y}^{m_{2}}\right)=\sum_{\beta=1}^{n_{p}} G_{0}\left(f_{x}^{m_{1}}, f_{y}^{m_{2}}\right) \exp \left(2 \pi i f_{x}^{m_{1}} \phi_{x}^{\beta}\right) \exp \left(2 \pi i f_{y}^{m_{2}} \phi_{y}^{\beta}\right)
$$

at frequencies given by

$$
f_{x}^{m_{1}}=\frac{m_{1}}{h_{1}}, \quad m_{1}=-\frac{M_{1}}{2}, \ldots, \frac{M_{1}}{2}, \quad f_{y}^{m_{2}}=\frac{m_{2}}{h_{2}}, \quad m_{2}=-\frac{M_{2}}{2}, \ldots, \frac{M_{2}}{2}
$$

and where $G_{0}\left(f_{x}^{m_{1}}, f_{y}^{m_{2}}\right)$ represents the fast Fourier transform of $g_{0}(x, y)$. The corresponding power spectral density can be obtained by equation (6). Frequencies with indices of $m_{1}$ and $m_{2}$ will always refer to those associated with the unit cell.

Note that the discrete power spectral densities of the complex and periodic structures are, in general, obtained at different frequencies. Furthermore, assuming that the dimensions of the unit cell are smaller than that of the random structure, the frequencies associated with the periodic structure will be spaced at larger intervals in comparison to the complex structure. Therefore, to directly compare the spectral density of each structure, the power spectral density of the complex structure has to be "rebinned" to match the frequencies of the periodic structure. For a frequency bin defined by 


$$
\frac{f_{x}^{m_{1}-1}+f_{x}^{m_{1}}}{2}<f_{x}<\frac{f_{x}^{m_{1}}+f_{x}^{m_{1}+1}}{2}, \quad \frac{f_{y}^{m_{2}-1}+f_{y}^{m_{2}}}{2}<f_{y}<\frac{f_{y}^{m_{2}}+f_{y}^{m_{2}+1}}{2}
$$

the rebinned power spectral density of the complex microstructure $\hat{P}^{c}\left(f_{x}^{m_{1}}, f_{y}^{m_{2}}\right)$ is written as

$$
\hat{P}^{c}\left(f_{x}^{m_{1}}, f_{y}^{m_{2}}\right)=\sum_{n_{2}=n_{2}^{\prime}}^{n_{2}^{\prime}} \sum_{n_{1}=n_{1}^{\prime}}^{n_{1}^{f}} w\left(n_{1}, n_{2}\right) P^{c}\left(f_{x}^{n_{1}}, f_{y}^{n_{2}}\right)
$$

where $n_{1}^{i} \leq n_{1} \leq n_{1}^{f}$ and $n_{2}^{i} \leq n_{2} \leq n_{2}^{f}$ are the range of indices whose frequency bins are within the intervals given in (10). Similarly, $w\left(n_{1}, n_{2}\right)$ represents the percentage of the frequency bin of $P^{c}\left(f_{x}^{n_{1}}, f_{y}^{n_{2}}\right)$ located in the intervals in (10).

For a given set of unit cell dimensions, the hole positions are found by minimizing the function

$$
\chi^{2}=\sum_{m_{2}=0}^{M_{2} / 2} \sum_{m_{1}=0}^{M_{1} / 2}\left[\frac{\hat{P}^{c}\left(f_{x}^{m_{1}}, f_{y}^{m_{2}}\right)-\left(A^{c} / A^{p}\right) P^{p}\left(f_{x}^{m_{1}}, f_{y}^{m_{2}}\right)}{\hat{P}^{c}\left(f_{x}^{m_{1}}, f_{y}^{m_{2}}\right)}\right]^{2}+\Phi\left(\phi_{x}^{1}, \ldots, \phi_{x}^{n p}, \phi_{y}^{1}, \ldots, \phi_{y}^{n p}\right)
$$

where $A^{c}$ and $A^{p}$ are the areas of the entire structure and the unit cell respectively. The function $\Phi$ becomes large when holes are either in contact or very close to one another, thereby providing an artificial penalty for solutions that are inconsistent with the form of the indicator function given in (7). Conversely, when all the holes are isolated from one another, $\Phi$ becomes identically zero. The specific form of the function $\Phi$ does not significantly affect the results.

Finding the absolute minimum of $\chi^{2}$ for a given set of cell dimensions was complicated by the fact that the residual $\chi^{2}$ typically has many local minima. Hole positions were found by initially considering the summation shown in equation (12) truncated to include only a few of the lowest frequencies, and minimizing this function. Higher frequency terms were then added in the summation, and the function was again minimized. This process was repeated until all of the terms in the summation were included. Each minimization was carried out by use of the conjugate gradient method [16]. Several initial guesses of the hole positions were required for each set of unit cell dimensions to be reasonably assured that a reasonable correlation between the spectral densities of each microstructure was obtained. With the current state of development of the numerical procedures, convergence to the absolute minimum of $\chi^{2}$ is not guaranteed. An 
incremental search was performed over all realistic unit cell dimensions by continually adjusting the aspect ratio $h_{2} / h_{1}$. The most representative unit cell had dimensions and hole positions corresponding to the smallest value of $\chi^{2}$ obtained by the procedures.

The motivation for using the power spectral density to compare the complex and periodic hole patterns is that it can be thought of as a probability density function in the frequency domain [17]. Periodic hole patterns with similar spectral densities to that of the actual perforated sheets should therefore have a comparable hole distributions, and presumably, similar mechanical behavior as well. A more intuitive way of thinking about the power spectral density is that it can be loosely thought of as representing the "diffraction pattern" of a two-phase material, in that the spectral density of the indicator function is mathematically very similar to the intensity distribution given by the kinematical theory of electron diffraction [18]. For example, if the hole distribution is highly ordered, the spectral density will have very sharp peaks, much like the diffraction pattern of a single crystal. Conversely, the spectral density becomes more diffuse as the distribution of holes becomes more random.

\subsection{Finite Element Calculations}

Simulations of uniaxial tensile tests were performed based on the selected unit cells and the results were compared to the experimental behaviors of the corresponding aluminum sheets. Finite element meshes were obtained using the commercial mesh generating program Hypermesh [19]. Finite strain, plane stress, four-node quadrilateral elements were used in all of the computations. Periodic boundary conditions were prescribed for each unit cell to ensure compatibility with surrounding unit cells and continuity of tractions across cell boundaries. Denoting the displacement and force at the upper right corner node by $\mathbf{u}^{\mathrm{p}}$ and $\mathbf{F}^{\mathrm{p}}$ respectively, the boundary conditions were prescribed as follows. For the four corners

$$
\begin{gathered}
u_{x}(0,0)=u_{x}\left(0, h_{2}\right)=u_{y}(0,0)=u_{y}\left(h_{1}, 0\right)=0 \\
u_{x}\left(h_{1}, h_{2}\right)=u_{x}^{p}, \quad u_{y}\left(h_{1}, h_{2}\right)=u_{y}^{p}
\end{gathered}
$$

For the nodes along the edges

$$
\begin{array}{lr}
u_{x}\left(x, h_{2}\right)-u_{x}(x, 0)=0, & u_{y}\left(x, h_{2}\right)-u_{y}(x, 0)=u_{y}^{p} \\
u_{x}\left(h_{1}, y\right)-u_{x}(0, y)=u_{x}^{p}, & u_{y}\left(h_{1}, y\right)-u_{y}(0, y)=0
\end{array}
$$


To simulate uniaxial tension, a nominal strain rate of $\dot{\varepsilon}_{y}=2.8 \times 10^{-4} s^{-1}$ was imposed in the vertical direction, and the net forces on the sides of the unit cell were assumed to vanish. These boundary conditions are imposed through the relations

$$
\dot{u}_{y}^{p}=h_{2} \dot{\varepsilon}_{y y}, \quad F_{x}^{p}=0
$$

The nominal stress-strain behavior of the unit cell is then given by

$$
\sigma_{y y}=\frac{F_{y}^{p}}{h_{1}}, \quad \varepsilon_{y y}=\frac{u_{y}^{p}}{h_{2}}
$$

assuming an initial unit thickness. In addition to satisfying compatible cell deformations, the boundary conditions described above also yield the necessary condition that the tractions, $\mathbf{T}$, across cell boundaries are continuous,

$$
\mathbf{T}(x, 0)=-\mathbf{T}\left(x, h_{2}\right), \quad \mathbf{T}(0, y)=-\mathbf{T}\left(h_{1}, y\right)
$$

The mechanical behavior of the aluminum was modeled as elastic-viscoplastic, with a stress ratestrain rate relation of the form

$$
\bar{\tau}=\mathbf{L}:\left(\mathbf{D}-\mathbf{D}^{p}\right)
$$

where $\frac{\nabla}{\tau}$ is the Jaumann rate of Kirchhoff stress, $\mathbf{L}$ is a tensor of isotropic elastic moduli, and $\mathbf{D}$ is the total rate of deformation. The plastic rate of deformation was assumed to be isotropic, powerlaw viscoplasticity of the form

$$
\mathbf{D}^{p}=\dot{\varepsilon}_{0}\left(\frac{\bar{\sigma}}{g(\bar{\varepsilon})}\right)^{m} \frac{3 \mathbf{S}}{2 \bar{\sigma}}
$$

where $\bar{\sigma}$ is the effective stress, $\bar{\varepsilon}$ is the effective plastic strain, and $\mathbf{S}$ is the deviatoric stress. The flow strength $g(\bar{\varepsilon})$ of the aluminum was assumed to be first strain hardening, and then strain softening 


$$
g(\bar{\varepsilon})= \begin{cases}\sigma_{y}+\left(\sigma_{\max }-\sigma_{y}\right)\left[1-\left(\frac{\bar{\varepsilon}-\varepsilon_{1}}{\varepsilon_{1}}\right)^{2}\right]^{n}, & \bar{\varepsilon} \leq \varepsilon_{1} \\ \frac{1}{2} \sigma_{\max }\left\{1+\cos \left[\pi\left(\frac{\bar{\varepsilon}-\varepsilon_{1}}{\varepsilon_{2}-\varepsilon_{1}}\right)\right]\right\}, & \varepsilon_{1}<\bar{\varepsilon}<\varepsilon_{2} \\ 0, & \bar{\varepsilon} \geq \varepsilon_{2}\end{cases}
$$

The above relations were chosen so that the material strain-hardens up to a plastic strain of $\varepsilon_{1}$, strain-softens at plastic strains between $\varepsilon_{1}$ and $\varepsilon_{2}$, and has a complete loss of load carrying capacity at plastic strains greater than $\varepsilon_{2}$. The initial flow strength is given by $\sigma_{y}$ while the maximum flow strength is indicated by $\sigma_{\max }$. The Young's modulus of the aluminum was also assumed to be a function of accumulated plastic strain

$$
E(\bar{\varepsilon})= \begin{cases}E_{0}, & \bar{\varepsilon} \leq \varepsilon_{1} \\ \frac{1}{2} E_{0}\left\{1+\cos \left[\pi\left(\frac{\bar{\varepsilon}-\varepsilon_{1}}{\varepsilon_{2}-\varepsilon_{1}}\right)\right]\right\}, & \varepsilon_{1}<\bar{\varepsilon}<\varepsilon_{2} \\ 0, & \bar{\varepsilon} \geq \varepsilon_{2}\end{cases}
$$

The addition of equation (23) to the model accounts for the reduction in stiffness that is expected as damage accumulates in the aluminum matrix. We incorporated the constitutive law into the commercial finite element code ABAQUS [20] through a user-supplied subroutine UMAT, using a rate-tangent integration method by Peirce et al. [21].

\subsection{Determination of Material Parameters}

The most obvious method for the determination of material parameters would be standard uniaxial tensile tests on (unperforated) aluminum sheets. After material softening and subsequent localization of deformation, however, the mechanical behavior of the aluminum can only be indirectly inferred through further analysis. The anticipated failure mechanism in the perforated aluminum sheets is the localization of deformation between adjacent holes. For this reason, we decided to test sheets with a uniform square array of holes, and to infer the mechanical properties of the aluminum by finite element analyses of a corresponding unit cell. It is hoped that the 
parameters determined in this manner will be most appropriate for describing the softening behavior of the aluminum in the simulations using the selected representative unit cells.

More specifically, the tensile sample used to determine the material properties had a $10 \times 10$ array of $1.016 \mathrm{~mm}$ holes. The holes were uniformly spaced over an area with dimensions $28.5 \mathrm{~mm} \times$ $28.5 \mathrm{~mm}$ to give a hole area fraction of 10 percent. The simulation of the experiment was performed with a unit cell having a single hole in its center, and applying the appropriate periodic boundary conditions. Because of the additional symmetries of this particular periodic structure, only one-quarter of the cell had to be analyzed.

Two levels of mesh refinement were used in the simulations of the selected representative cells with twelve holes; the mesh of the simple unit cell used to ascertain material constants had approximately the same level of refinement as the coarser mesh used for the representative cells.

As in Becker and Smelser [10], the stress exponent was taken to be $m=100$, while the material parameters $\sigma_{y}, \sigma_{\max }, \varepsilon_{1}, \varepsilon_{2}$, and $n$ were adjusted to provide a fit to the experimental data. The experimental and numerical stress-strain curves are shown in Figure 2. With the level of discretization used in the calculations, the overall rate in which the stresses declined after maximum load was surprisingly insensitive to the value of the failure strain $\varepsilon_{2}$. Even when a value of $\varepsilon_{2}$ only slightly greater than $\varepsilon_{1}$ was used, the numerical simulations exhibited a significantly slower drop-off in stresses after maximum load than was observed in the experiments. One potential cause of this discrepancy is that fracture in the experiments occurs across only one row of holes, while the calculations implicitly assume that failure would occur across every row in the array of holes. The value of the failure strain was somewhat arbitrarily chosen to be $\varepsilon_{2}=0.66$, simply because relatively large values of $\varepsilon_{2}$ tended to increase the stable time step increment used in the computations. The failure mechanism exhibited in both the experiments and the simulations was strain localization directly across the ligaments between holes. The specific material parameters used to model the aluminum matrix are shown in Table I. 
Table I. Material Properties of aluminum 1100-H14.

\begin{tabular}{|l|l|}
\hline \multicolumn{1}{|c|}{ Properties } & \multicolumn{2}{|c|}{ Value } \\
\hline Initial Young's Modulus, $E_{0}$ & $72000 \mathrm{MPa}$ \\
\hline Poisson's Ratio, $v$ & 0.3 \\
\hline Yield Strength, $\sigma_{Y}$ & $9 \mathrm{MPa}$ \\
\hline Ultimate Strength, $\sigma_{\max }$ & $100 \mathrm{MPa}$ \\
\hline Softening Strain, $\varepsilon_{1}$ & 0.36 \\
\hline Failure Strain, $\varepsilon_{2}$ & 0.66 \\
\hline Stress Exponent, $m$ & 100 \\
\hline Strain Rate, $\dot{\varepsilon}_{0}$ & $0.00028 \mathrm{~s}^{-1}$ \\
\hline Strain Hardening Parameter, $n$ & 0.56 \\
\hline
\end{tabular}

\section{Results}

The six complex patterns used in the experiments and the corresponding unit cells used in the finite element calculations are shown in Figure 3. In each case, the periodic hole patterns appear to have a similar spatial distribution as complex structures from which they were derived. The unit cells used for the finite element calculations are outlined in the lower left corner of the periodic structures. An example of the power spectral densities for the complex and periodic patterns is given in Figure 4.

Photographs of the tensile specimens, both before and after testing, are shown in Figure 5. During the early stages of loading, visible, localized deformation occurred around all of the holes. Thin ligaments between some of the holes subsequently necked and then failed. Upon further deformation, larger ligaments between holes necked primarily along the horizontal direction. The first major drop in load corresponded to rupture of a number of these ligaments, often extending to one edge of each of the specimens. 
Modeling localization and failure using the finite element method introduces a complicating factor into the analysis. Unfortunately, once localization of deformation occurs, the presence of the finite element mesh introduces an artificial length scale into the problem which in turn causes the solution to become mesh dependant. We therefore performed calculations on our representative unit cells with different element densities to ascertain the effects of mesh refinement on the numerical solutions. Each unit cell was therefore modeled with meshes of approximately 900 and 1800 plane stress, four node, quadrilateral elements.

Figures 6 compares the experimentally observed nominal stress-strain behavior with those predicted by the finite element calculations using the representative unit cells. Figure 6(a) shows the results from tensile experiments on perforated aluminum sheets. Figure $6(\mathrm{~b})$ shows results for meshes with approximately 900 elements while Figure 6(c) corresponds to calculations with about 1800 elements. In almost every case, the figures show that the model calculations exhibit the same trends in strength and ductility as the six different hole patterns, although the calculations overestimate the ductility of the sheets by a significant margin in all cases. In one case, however (microstructure \#4), there is a significant discrepancy between the ductility predicted by the two different meshes.

Figure 7 displays contour plots of effective plastic strain just prior to failure in each of the representative unit cells modeled with approximately 1800 elements. In each case, the predicted failure path corresponds to the regions of maximum plastic strain. The effect of the periodic boundary conditions is evident, particularly for pattern \#1, where the failure path meanders from the top to the bottom of the unit cell. The density of the finite element mesh used in the calculations is also clearly visible in the figures. Figure 8 compares contours of plastic strain for the microstructure \#4, the case where the different mesh densities gave significantly different predictions of sheet ductility. In this particular case, competing failure paths developed in the analysis with the finer mesh, which in turn resulted in a significant increase in the predicted ductility when compared with the coarse mesh.

In order to more clearly see the trends exhibited in the experiments and by the corresponding models, we also compared values of the relative ultimate strengths in Figure 9. In Figure 9, the 
experimental strengths have all been normalized by the measured ultimate strength of hole pattern \#1. In contrast, the predicted strengths have been normalized to provide a "best fit" to the trends measured experimentally. From this perspective, the model also provides reasonably good estimates of the trends in ultimate strength exhibited by the perforated sheets.

\section{Discussion}

In this investigation, idealized two-phase microstructures were constructed by perforating sheets of aluminum with random patterns of holes. They were then subjected to uniaxial tension tests so that nominal stress-strain relationships could be obtained. Each of the complex hole patterns were modeled using representative unit cells with a statistically similar distribution of holes. The unit cells were obtained by comparing the power spectral densities of the image of the original random hole pattern with that of an idealized periodic pattern [13]. The resultant boundary value problem was solved by use of finite elements. The experimental results show the strong effect that hole distribution can have in thin sheets, which is also expected in other composite materials. The predicted nominal stress-strain curves follow the trends in ductility variations between the various microstructures, although an over-prediction of ductility is consistently observed.

There are a number of possible explanations as to why the model overestimated the observed ductility in every case. First, the calculations prescribe periodic boundary conditions on the representative unit cell, so that the effect of free edges on the behavior of the actual tensile specimens is not accounted for in the analysis. The presence of edges in these finite-width specimens may allow for premature failure of ligaments near the edges so that the observed ductility is lower in comparison to an infinitely large specimen. Second, the material model and parameters used in the calculations are highly simplified and may not accurately represent the behavior of the aluminum. A third possible reason is that failure will occur along the weakest of all possible failure paths in the test specimen. The chosen periodic structure, however, is only in some sense typical of the actual distribution of holes in the aluminum test specimens. The unit cell used in the finite element calculations is therefore unlikely to have a potential failure path that is as weak as the path observed experimentally. The final and most likely reason for the discrepancies, however, is simply that failure in the model will occur, by definition, across every unit cell that 
make up the periodic hole pattern. The fact that the model has, in essence, multiple failure paths leads to an over prediction of the ductility of the perforated sheets.

This idea can also be used to explain the differences in the ductility predicted by the different levels of mesh refinement for microstructure \#4. In the analysis with the finer mesh, an additional potential fracture path developed that significantly increased the predicted ductility. This result suggests that ductility predictions may be quite sensitive to small variations in unit cell geometry and material constitutive behavior, as well as the level of mesh refinement. In contrast, we expect that predictions of ultimate strength (and deformation behavior prior to localization) would be relatively insensitive to small variations in model parameters.

It is worth noting some other important limitations in the methods described in this paper. One obvious limitation is that the method is restricted to two-dimensional microstructures, while composites with short fiber or particle reinforcements are clearly three-dimensional in nature. Even for three-dimensional microstructures, however, it may be possible to adequately model the composite with a two-dimensional, generalized plain strain calculations. A more significant limitation in our view, however, is that the many composite microstructures (see, for example, Park et al. [22]) have regions with large clusters of particles, and conversely, regions with little or no reinforcement. Such a microstructure would require a unit cell with much larger numbers of reinforcements than those used in the present study, which could in turn render the associated finite element calculations overly complex and computationally intractable.

The numerical methods used in this study could also be improved upon. For example, for composites that have particles of various size, morphology, and orientation, it is presently unclear as to how representative reinforcements should be selected. Moreover, there are very likely better ways to compare the spectral densities of the complex and periodic microstructures, and better ways to find the minimum of the residual function $\chi^{2}$ (equation (12)) to ensure that the absolute minimum (and therefore the most representative unit cell) is found. Perhaps techniques used in image analysis or pattern recognition could be used to improve upon or even supplant the numerical methods used here. 
Despite the limitations outlined above, however, we believe that the central ideas of this study have been validated. With our experiments, we have shown that the spatial distribution of a second phase can significantly affect the mechanical behavior, particularly regarding the evolution of damage and eventual failure of the material. Using representative unit cells that have a statistically similar distribution of holes to that of the perforated aluminum sheets, we have successfully predicted the effects that the hole distribution had on the behavior of the sheets.

\section{Acknowledgements}

The authors are pleased to acknowledgement financial support provided by the Air Force Office of Scientific Research, contract number F49620-96-1-0198.

\section{References}

1. Lewandowski, J.J., Liu, C. and Hunt, W. H., Mater. Sci. Engrg., 1989, A107, 241.

2. Bao, G., Hutchinson, J. W. and McMeeking, R. M. , Acta Metall. Mater., 1991, 39, 1871.

3. Christman, T., Needleman, A. and Suresh, S. , Acta Metall., 1989, 37, 3029.

4. Koplik, J. and Needleman, A., International Journal of Solids \& Structures., 1988, v24 n8, 835

5. Needleman, A., J. appl. Mech., 1987, 54, 525.

6. Finot, M., Shen, Y-L, Needleman, A., and Suresh, S., Metall. Mater. Trans., 1994, A25, 2403.

7. Llorca, J., Needleman, A. and Suresh, S., Acta Metall. Mater., 1991, 39, 2317.

8. Povirk, G. L., Stout, M. G., Bourke, M., Goldstone, J., Lawson, A. C., Lovato, M., MacEwen, S. R., Nutt, S. R. and Needleman, A., Acta Metall. Mater., 1992, 40, 2391.

9. Magnuson, P. E., Dubensky, E. M. and Koss, D. A., Acta Metall., 1988, 36, 1503.

10. Becker, R. and Smelser, R. E., J. Mech. Phys. Solids, 1994, 42, 773.

11. Ostoja-Starzewski, M., Sheng, P. Y. and Jasiuk, I., J. Eng. Mater. Tech., 1994, 116, 384.

12. Moorthy, S., and Ghosh, S., Comput. Methods Appl. Mech. Engrg., 1998, 151, 377.

13. Povirk, G. L., Acta Metall. Mater., 1995, 43, 3199.

14. Baker, H. et al., eds., Metals Handbook, vol. 2 (Metals Park, OH: American Society for Metals), 1979, 29.

15. LabVIEW ${ }^{\mathrm{TM}}$ version 4.0 (National Instruments Corporation 1996, www.ni.com)

16. Press, W.H., Teukolsky, S.A., Vetterling, W.T. and Flannery, B.P. Numerical Recipes in Fortran (Cambridge, U.K.: Cambridge University Press), 1992. 
17. Parzen, E., Stochastic Processes (Holden-Day, San Francisco, California), 1962, 110.

18. Hirsch, P. B., Howie, A., Nicholson, R. B., Pashley, D. W. and Whelan, M. J., Electron Microscopy of Thin Crystals (2 ${ }^{\text {nd }}$ Edition, Kreiger, Malabar, Florida), 1977.

19. HyperMesh ${ }^{\mathrm{TM}}$ version 2.1(Altair Computing, Inc. 1997, www.altair.com)

20. ABAQUS ${ }^{\mathrm{TM}}$ version 5.7 (Hibbitt, Karlsson \& Sorensen, Inc. 1997, www.hks.com)

21. Peirce, D., Shih, C. F. and Needleman, A., Comp. Struct., 1984, 18, 875.

22. Park, J., Lenshek, D. X., and Povirk, G. L., Acta Mater., 1997, 45, 1351. 


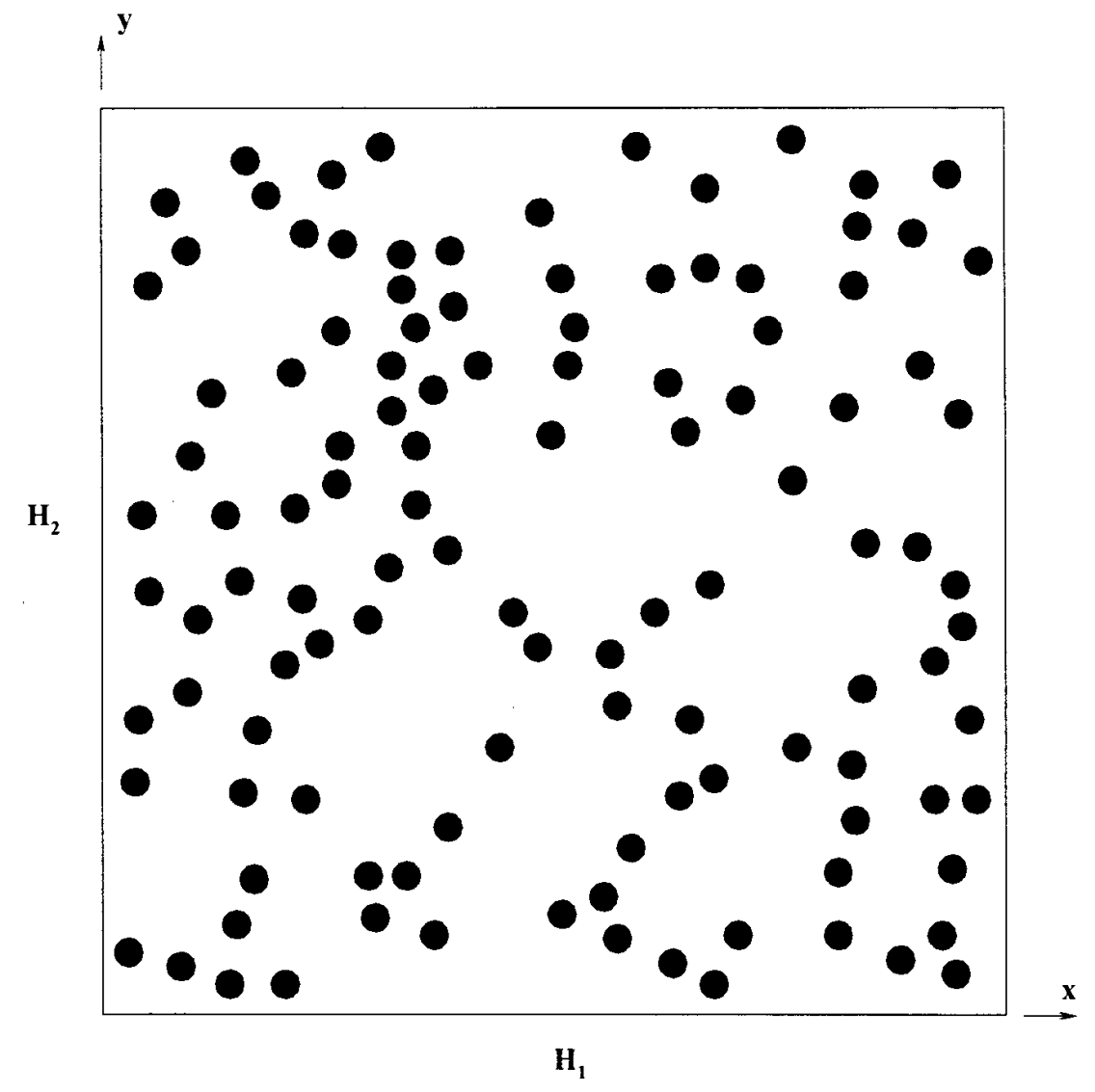

Figure 1. Typical hole pattern used for the perforated aluminum sheets. 


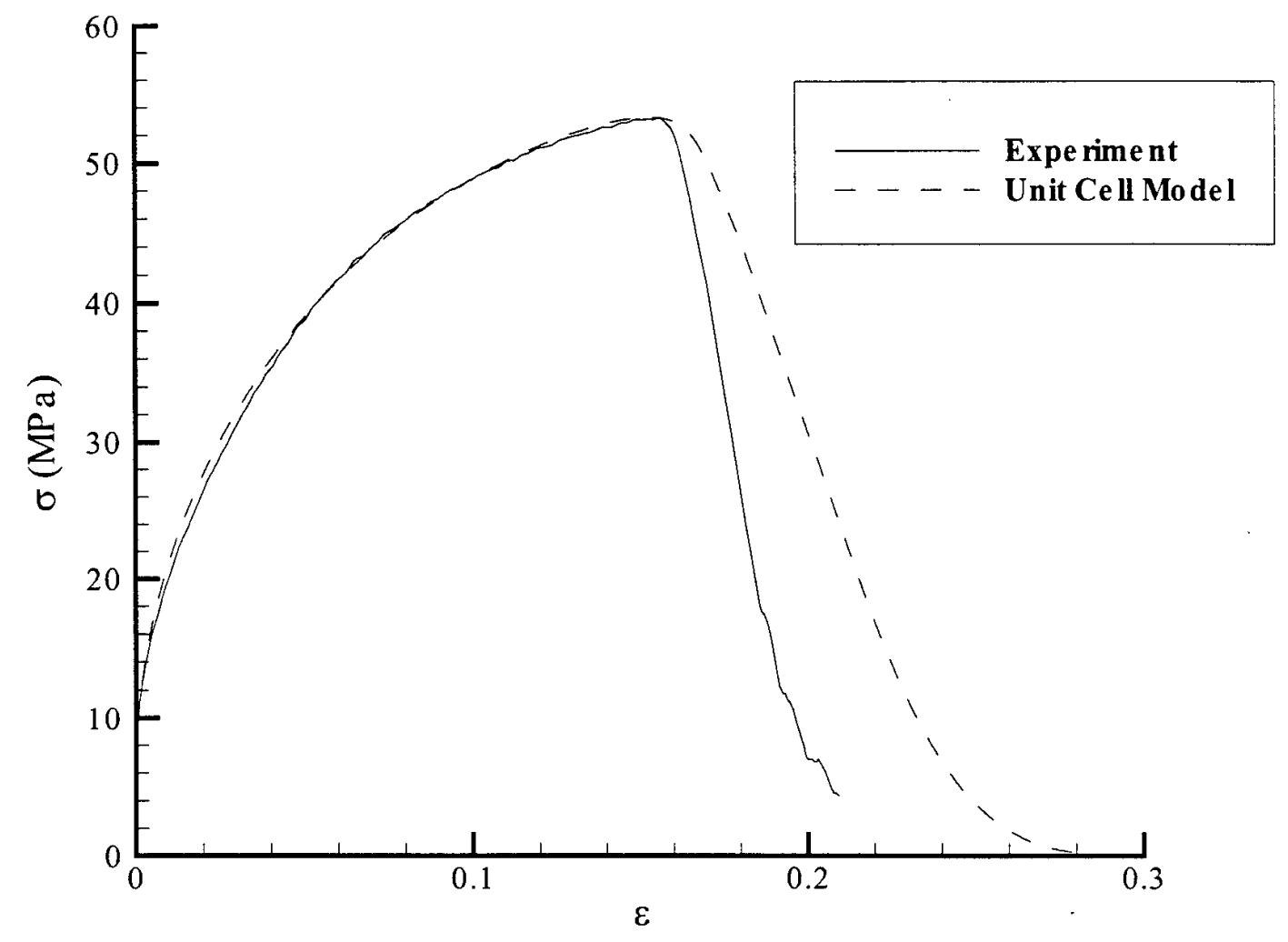

Figure 2. The solid line represents the nominal stress vs. strain curve for a perforated aluminum sheet with a simple cubic pattern of holes and a volume fraction of ten percent. The dashed line is the corresponding predicted response of a simple unit cell using the material properties listed in Table I. 
Perforated Sheet Microstructure

Periodic Structure
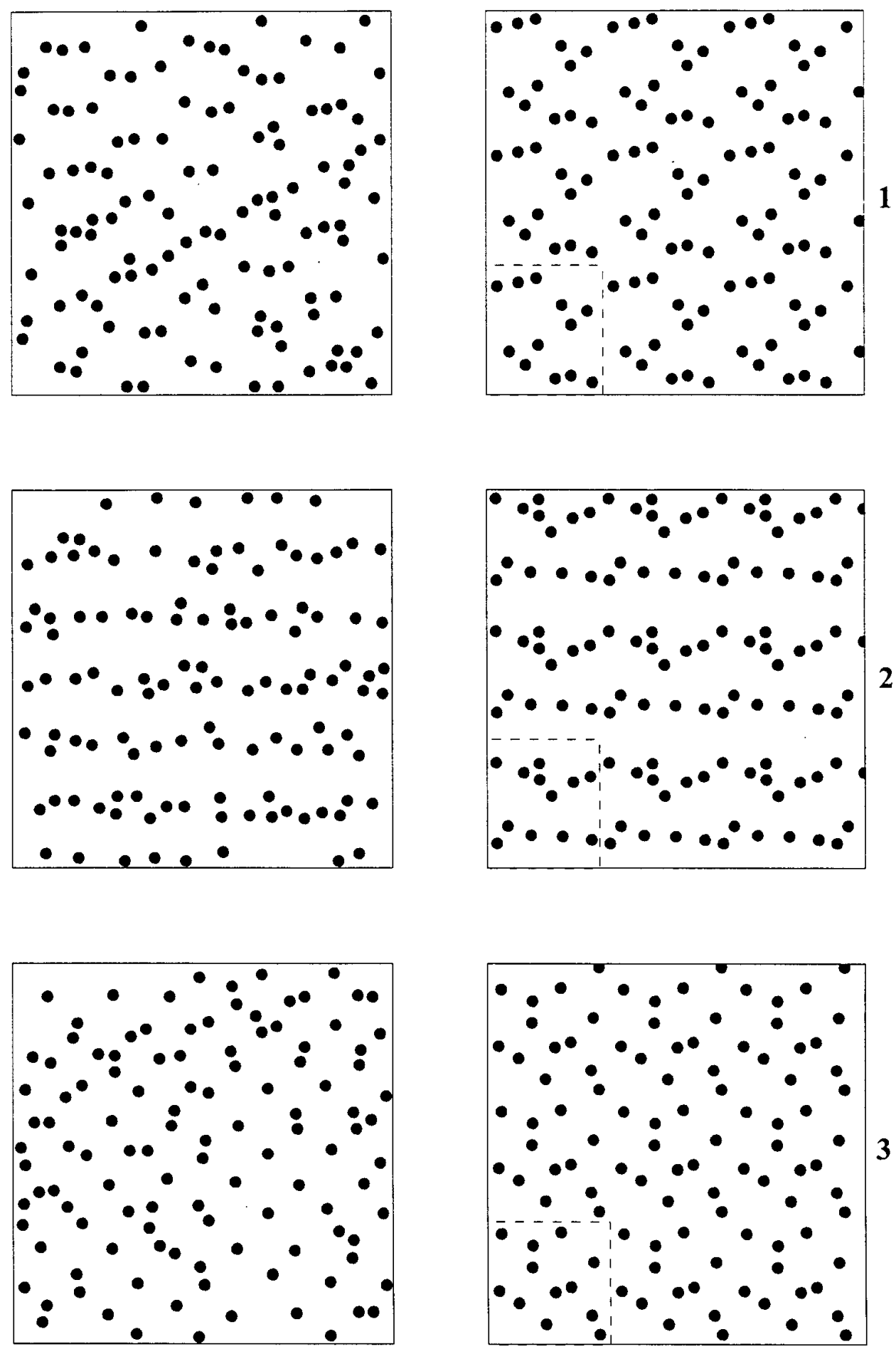

Figure 3. Diagrams of the 6 microstructures used in perforated aluminum sheets (left) and their corresponding periodic structures (right). The unit cells used in finite element calculations are indicated by dashed rectangles. 

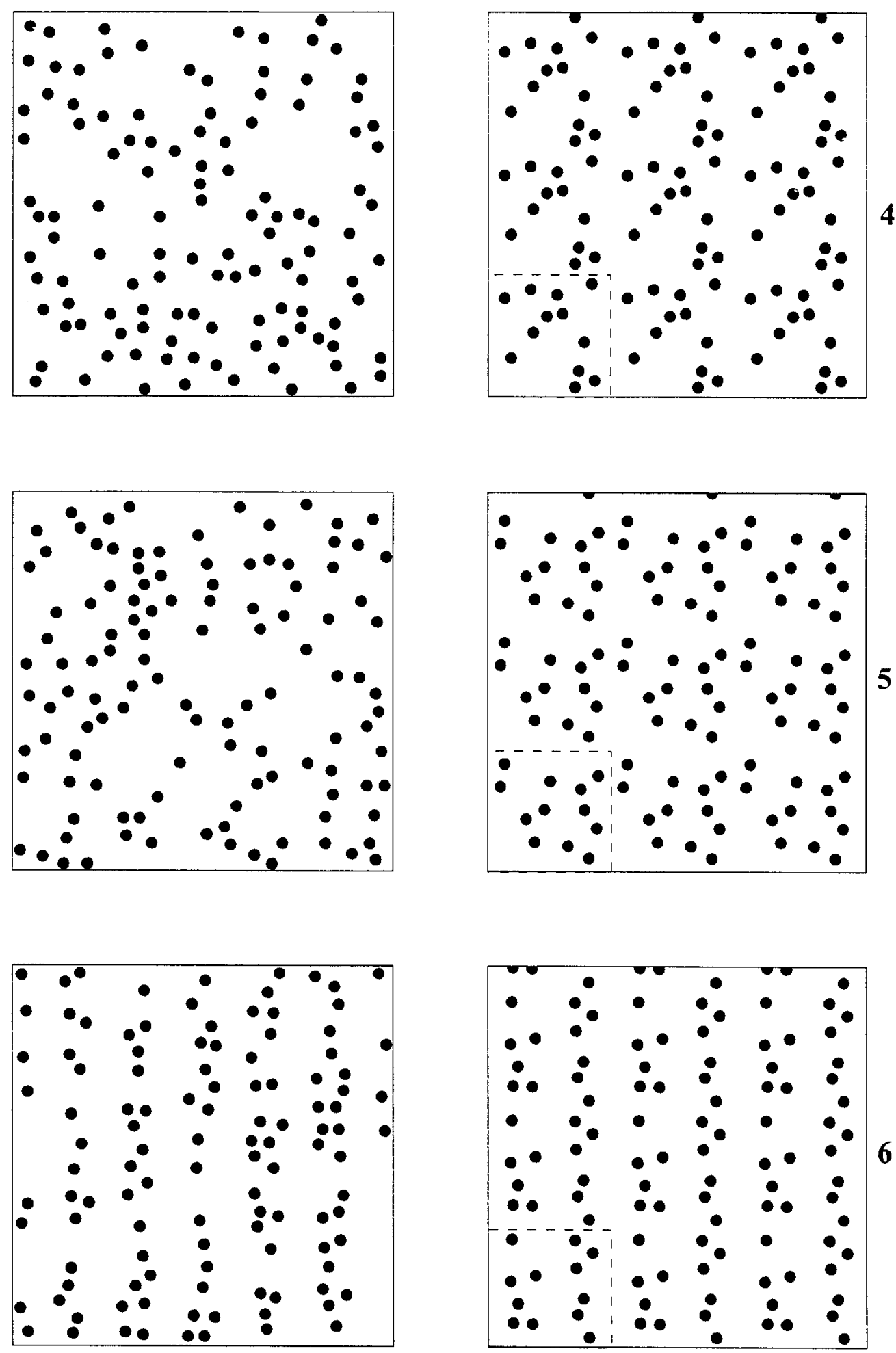

Figure 3. (continued) 

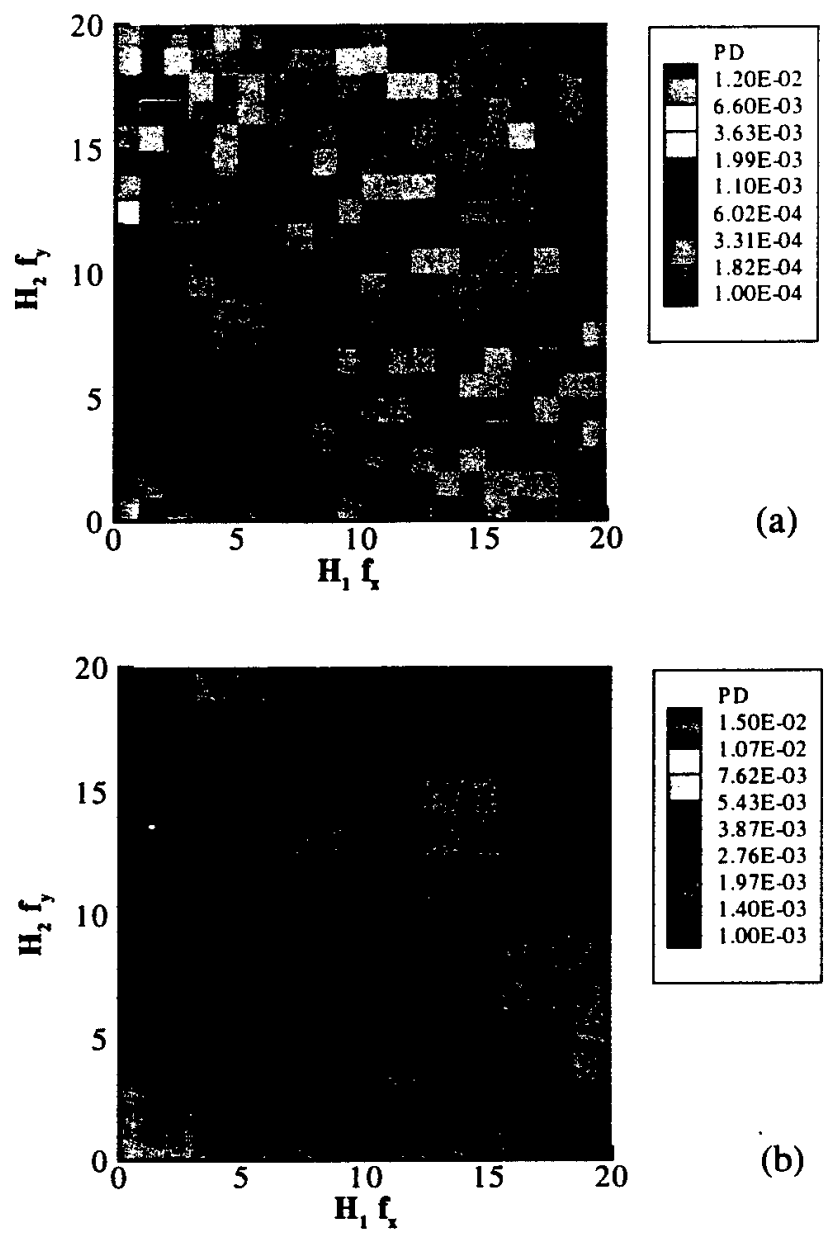

(b)
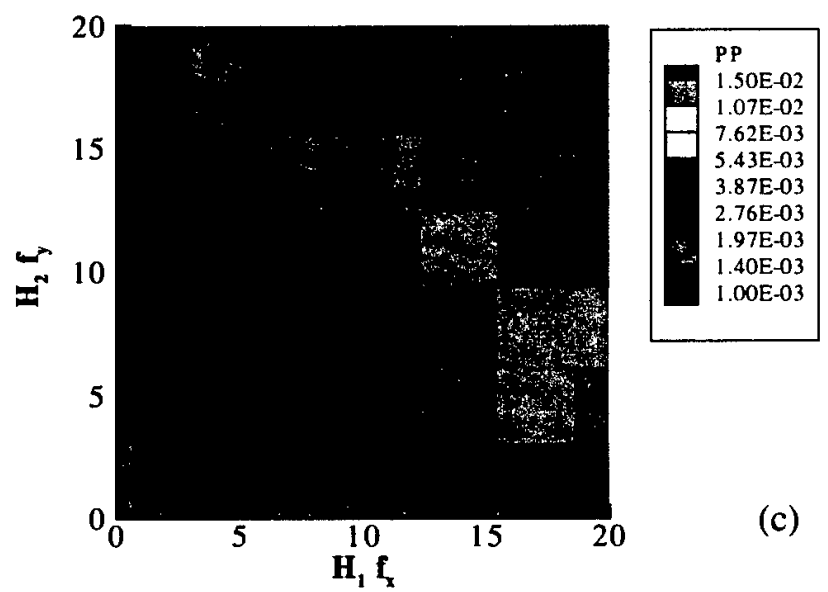

(c)

Figure 4. Power spectral densities of (a) the original hole pattern; (b) the original hole pattern after "rebinning"; and (c) the corresponding periodic structure. 

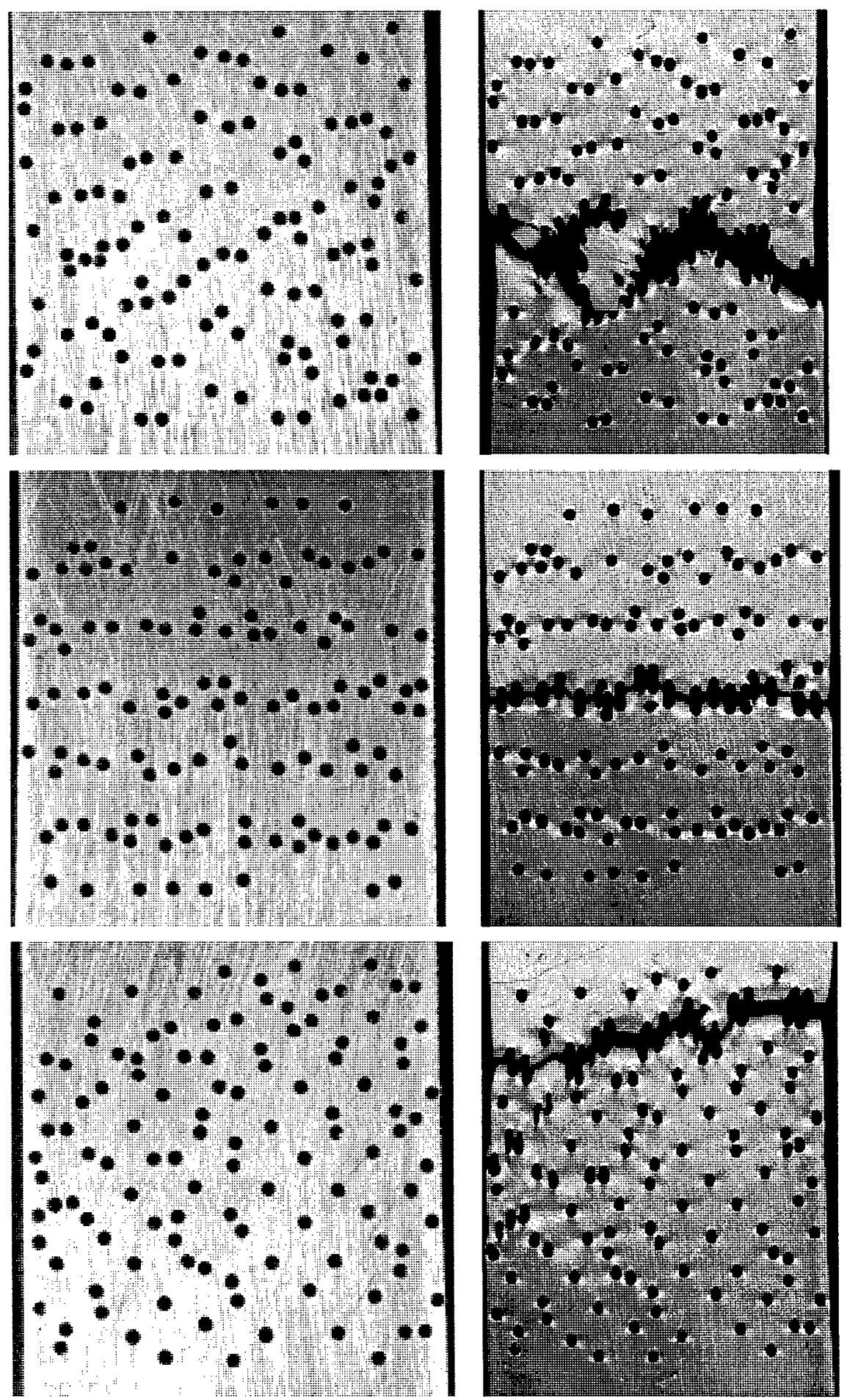

Figure 5. Photographs of the perforated aluminum sheets before and after the uniaxial tensile experiments. 
Before tensile experiment
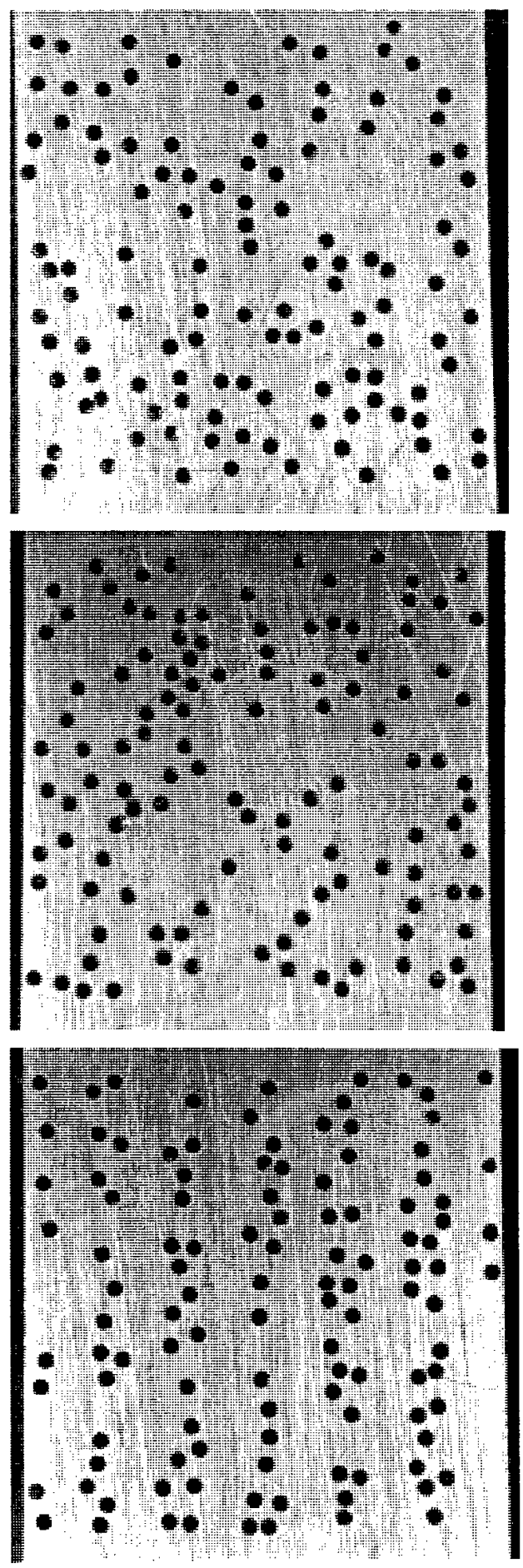

After tensile experiment
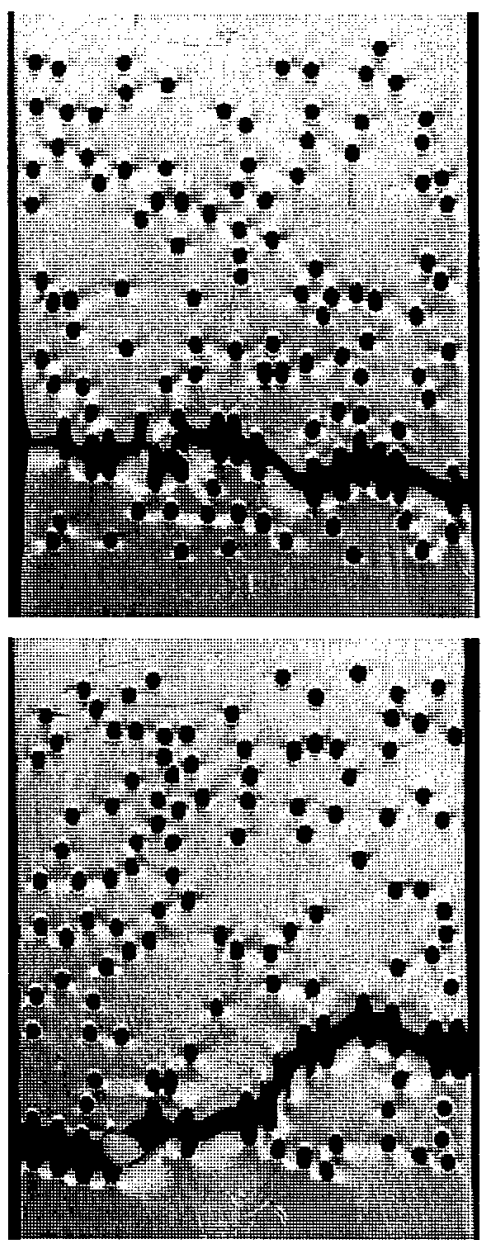

Figure 5. (continued) 


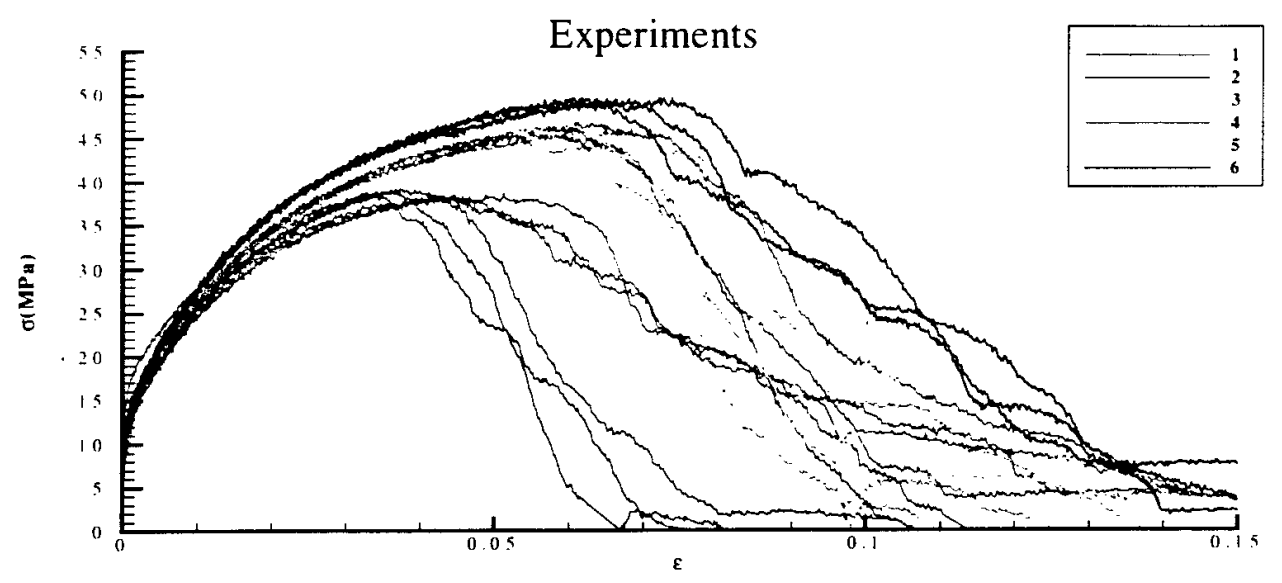

(a)

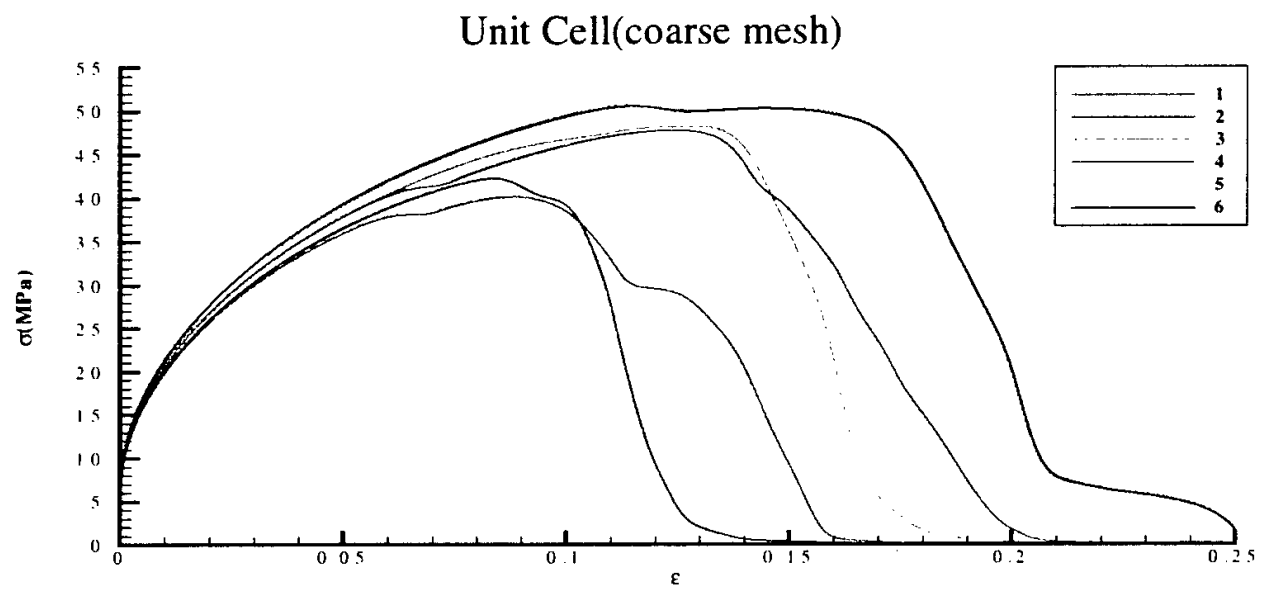

(b)

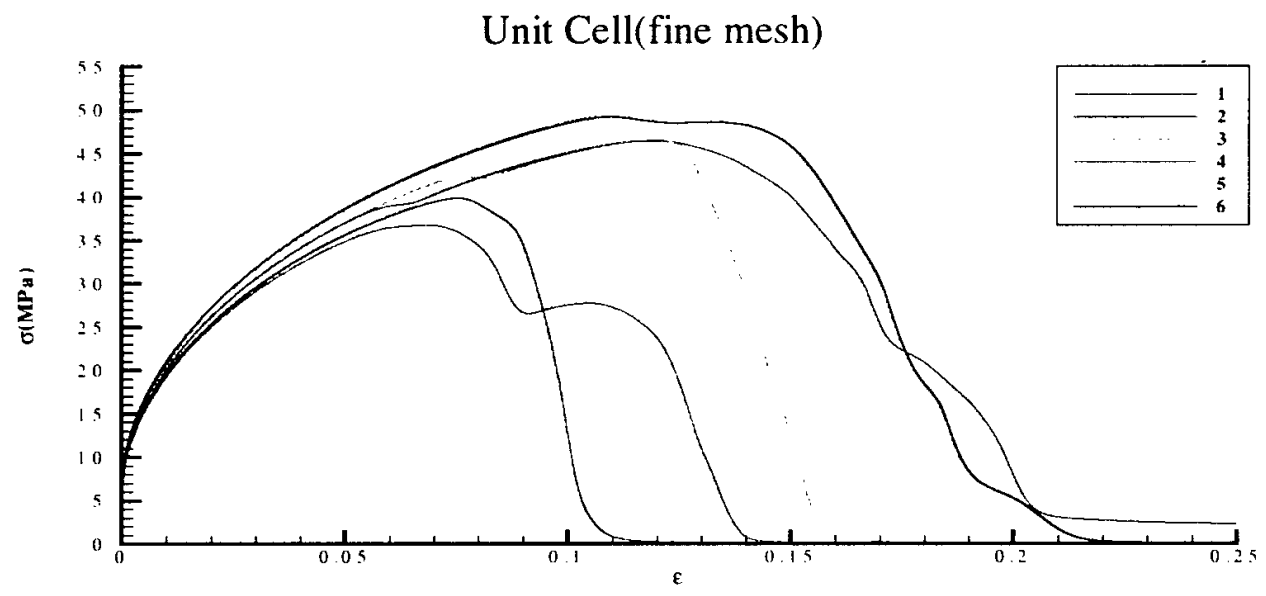

(c)

Figure 6. Stress vs. strain behavior of (a) the perforated aluminum sheets; (b) the corresponding unit cell calculations with approximately 900 elements; (c) unit cell calculations with approximately 1800 elements. Each color represents a different hole pattern; each hole pattern was tested three times experimentally. 

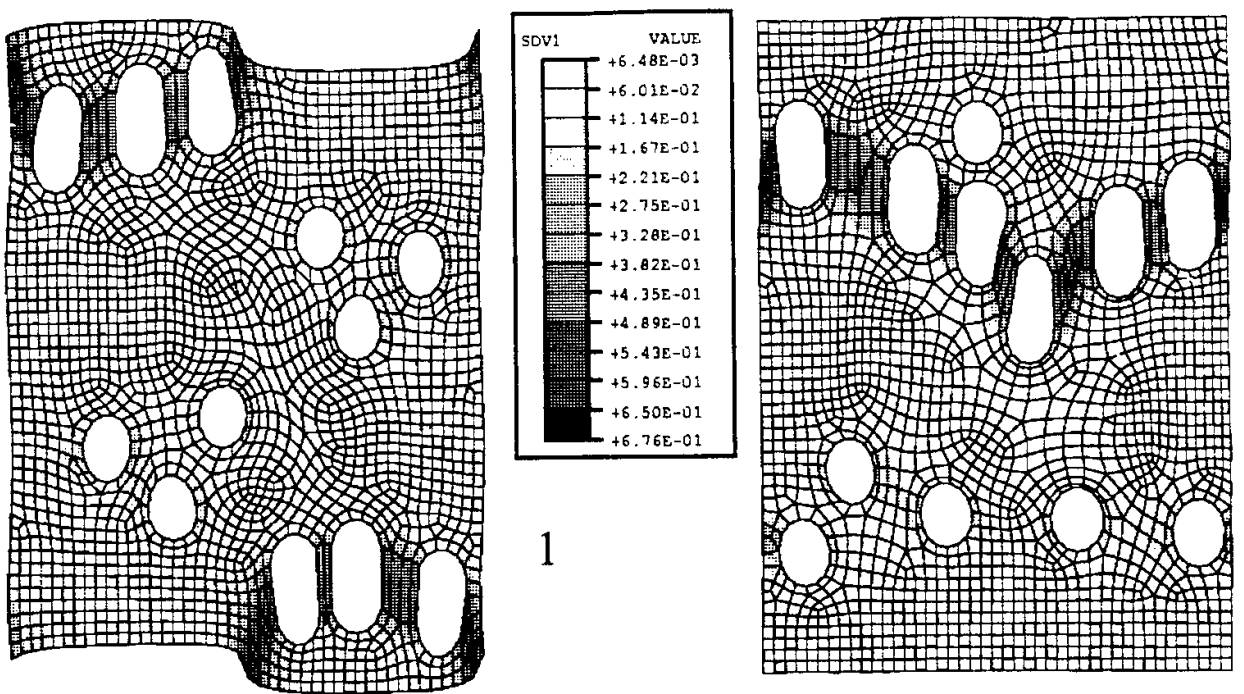

2
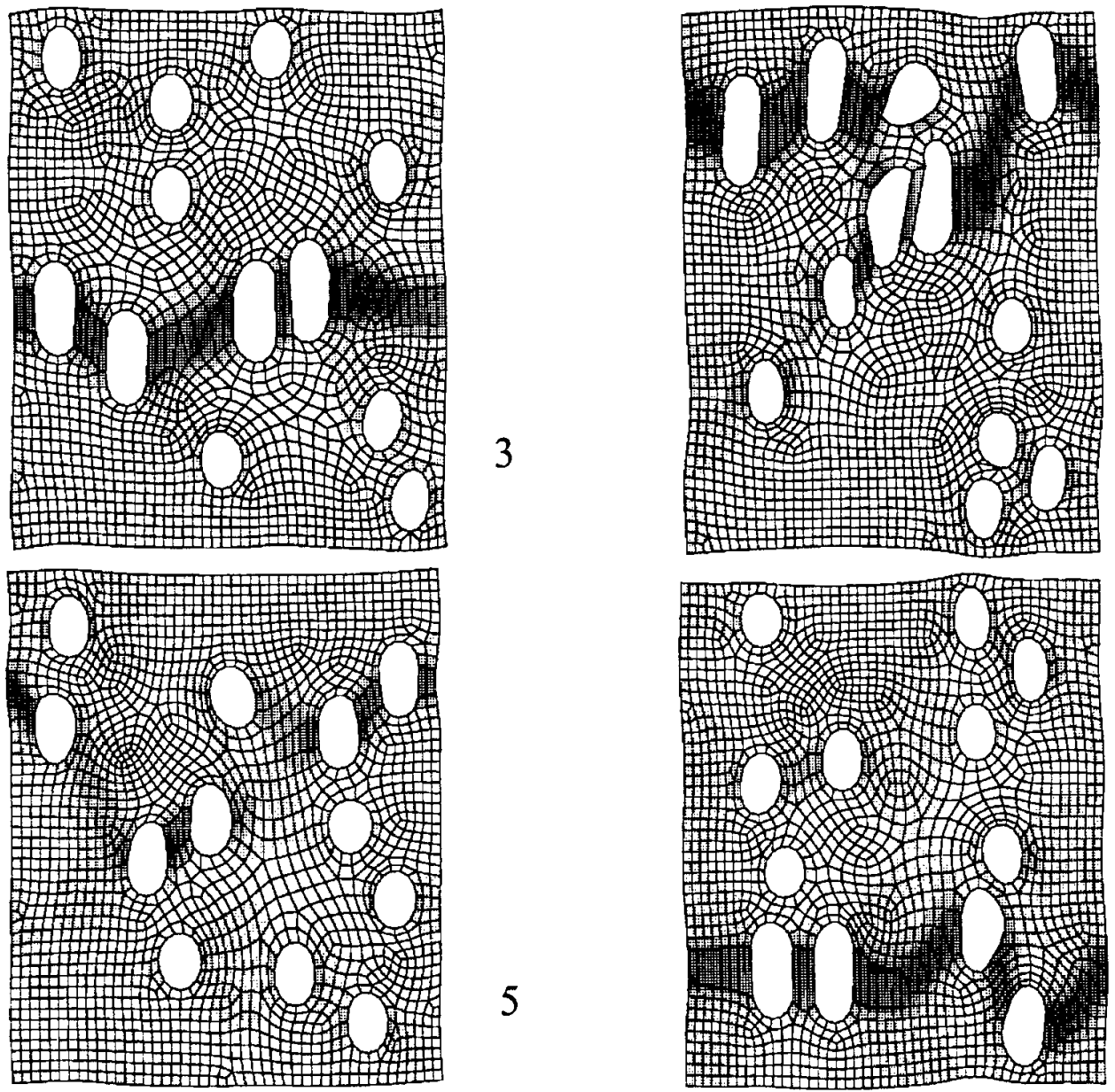

Figure 7. Contour plots of accumulated effective plastic strain for representative unit cells. Regions of dark shading indicate elements that have completely failed. 

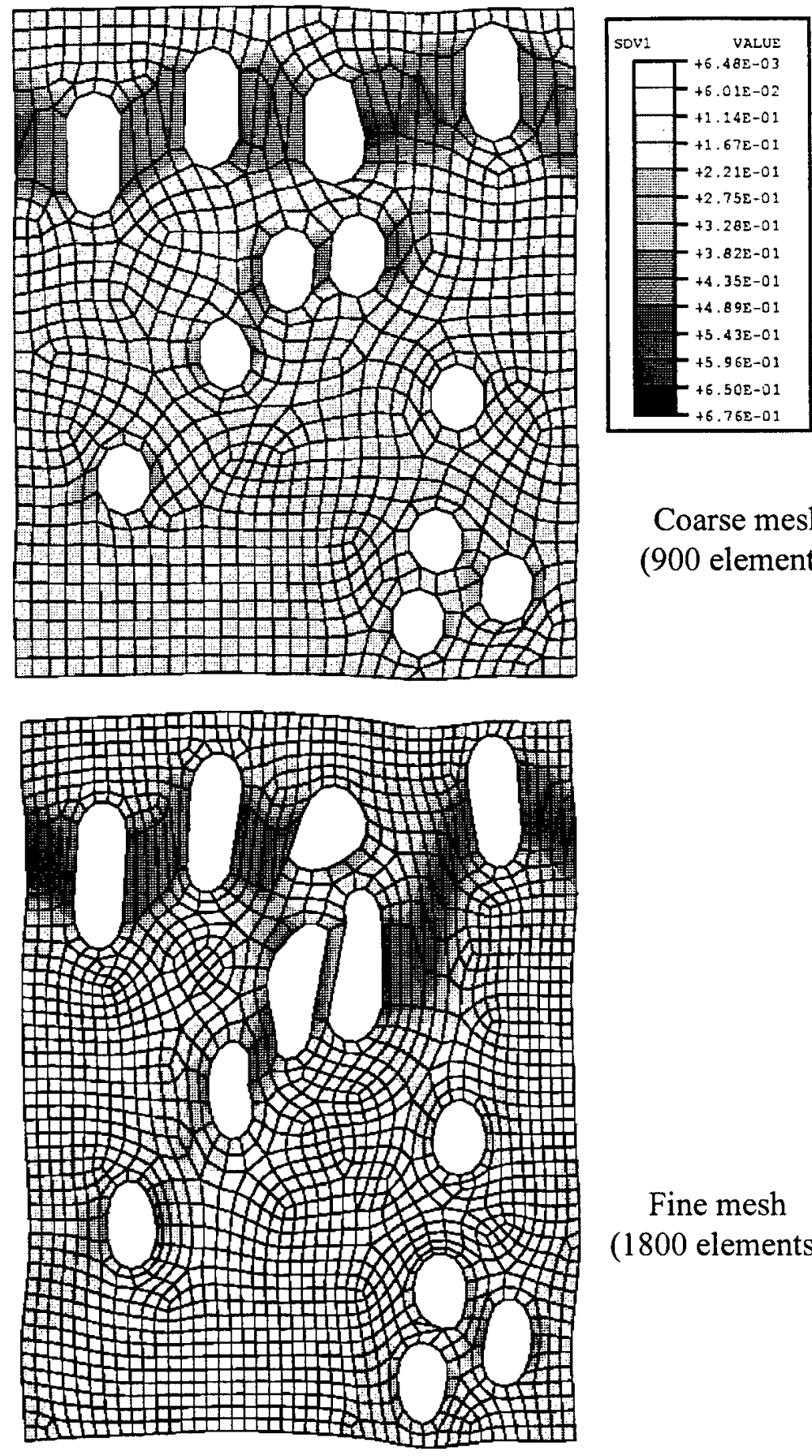

Coarse mesh

(900 elements)

Fine mesh

(1800 elements)

Figure 8. Contour plots of effective plastic strain of unit cell \#4 with coarse and fine mesh. For this particular unit cell, competing failure paths develop with the fine mesh, which in turn causes an increase in predicted ductility. 


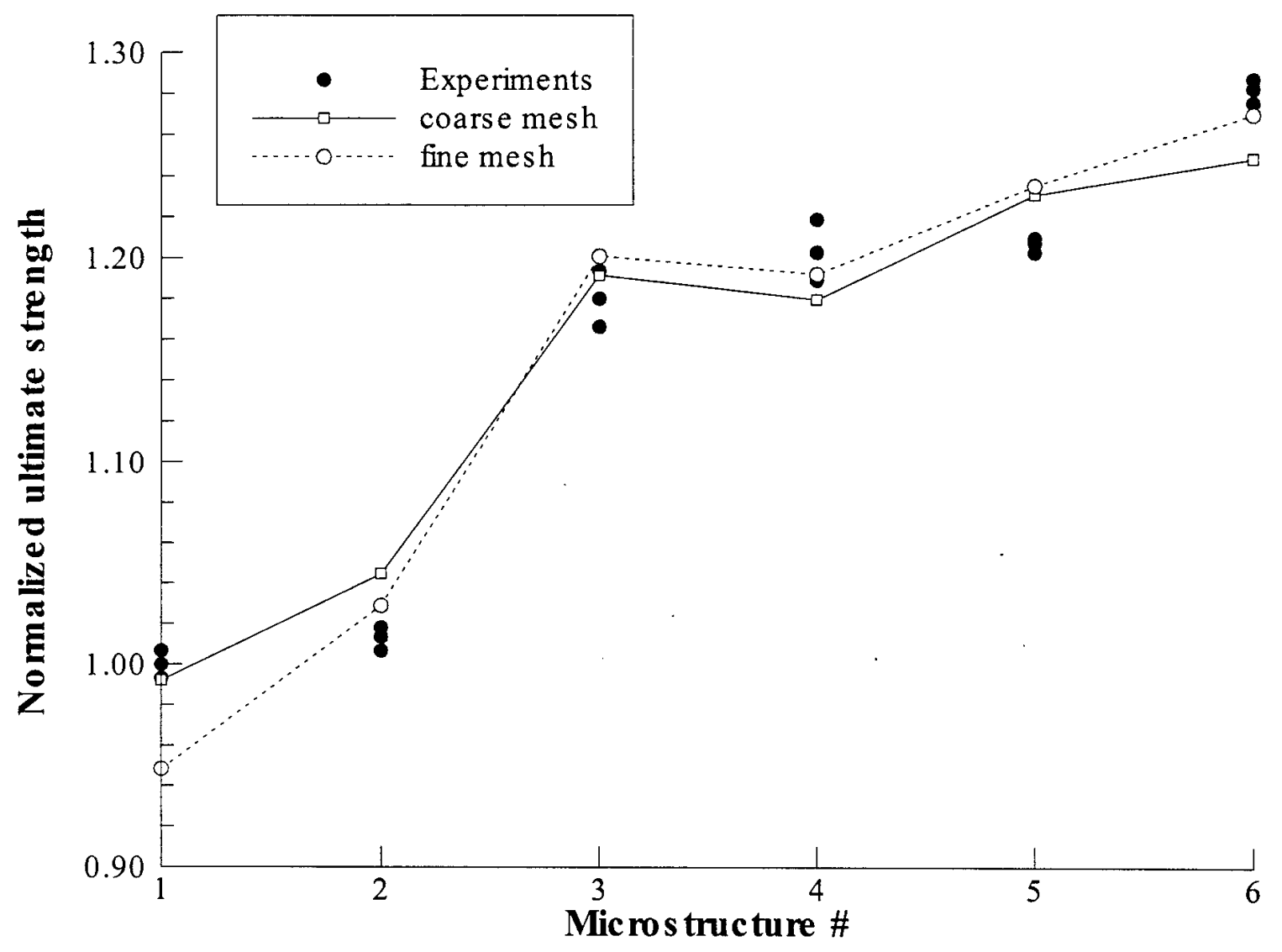

Figure 9. Normalized ultimate strengths of the perforated aluminum sheets compared to the strengths predicted by the unit cell calculations. 